\title{
A systematic search for novae in M 31 on a large set of digitized archival Schmidt plates ${ }^{\star}$
}

\author{
M. Henze ${ }^{1}$, H. Meusinger ${ }^{1}$, and W. Pietsch ${ }^{2}$ \\ 1 Thüringer Landessternwarte Tautenburg, 07778 Tautenburg, Germany \\ e-mail: henze@tls-tautenburg.de \\ 2 Max-Planck-Institut für extraterrestrische Physik, 85748 Garching, Germany
}

Received 16 July 2007 / Accepted 14 September 2007

\section{ABSTRACT}

\begin{abstract}
Aims. This paper reports on the detection of optical novae in our neighbour galaxy M 31 based on digitized historical Tautenburg Schmidt plates. The accurate positions of the detected novae lead to a much larger database when searching for recurrent novae in M31.

Methods. We conducted a systematic search for novae on 306 digitized Tautenburg Schmidt plates covering a time span of 36 years from 1960 to 1996 . From the database of both $\sim 3 \times 10^{5}$ light curves and $\gtrsim 10^{6}$ detections on only one plate per colour band, nova candidates were efficiently selected by automated algorithms and subsequently individually inspected by eye.

Results. We report the detection of 84 nova candidates. We found 55 nova candidates from the automated analysis of the light curves. Among these, 22 were previously unknown, 12 were known but not identified on Tautenburg Schmidt plates before, and 21 novae had been discovered previously on Tautenburg plates. An additional 29 known novae could be confirmed by the detailed investigation of single detections. One of our newly discovered nova candidates shows a high position coincidence with a nova detected about 30 years earlier. Therefore, this object is likely to be a recurrent nova. Furthermore, we re-investigated all 41 nova candidates previously found on Tautenburg plates and confirm all but two. Positions are given for all nova candidates with a typical accuracy of $\sim 0$ ". 4 . We present light curves and finding charts as online material.

Conclusions. The analysis of the plates has shown the wealth of information still buried in old plate archives. Extrapolating from this survey, digitization of other historical M 31 plate archives (e.g. from the Mount Wilson or Asiago observatories) for a systematic nova search looks very promising.
\end{abstract}

Key words. galaxies: individual: M 31 - novae, cataclysmic variables - catalogs

\section{Introduction}

Classical novae $(\mathrm{CN})$ are thermonuclear explosions on the surface of white dwarfs (WD) in cataclysmic binary systems. These explosions are caused by the transfer of matter from the companion to the WD. The transferred hydrogen-rich matter is accumulated on the surface of the WD until hydrogen ignition starts as a thermonuclear runaway process in the degenerated matter of the envelope. The resulting expansion of the hot envelope causes the luminosity of the star to rise by more than 9 mag within a few days (see Hernanz 2005; Warner 1995, and references therein).

A comprehensive compilation of novae in nearby galaxies is desirable for various issues such as nova physics (e.g., Pietsch et al. 2005) or the distributions of stellar populations (e.g., Ciardullo et al. 1987; Hatano et al. 1997; Yungelson et al. 1997). In our galaxy, the investigation of the nova population is hampered by the large area (namely the whole sky) to be scrutinised and by our unfavourable position close to the Galactic Plane. For nearby extragalactic systems, the situation is comparatively more promising. In particular, our huge neighbour galaxy M31 is very suitable for different kinds of statistical studies of novae because of its proximity and the high number of optical nova outbursts per year.

* Tables 10-19 and Figs. 8-14 are only available in electronic form at http://www . aanda.org
Pietsch et al. (2005) find that optical novae represent the major class of supersoft X-ray sources (SSS) in M31. More than $30 \%$ of the optical novae in the M31 centre area show an SSS phase within a year (Pietsch et al. 2007, hereafter PHS07). The X-ray monitoring of optical novae allows the mass of the ejecta, the burned mass, and the mass of the WD in the system to be constrained.

There have been various systematical surveys of novae in M31 for more than 80 years starting with the pioneering work of Hubble (1929) and Arp (1956). Among the many other contributors, we refer here only to the work of Rosino et al. (1964, 1973, 1989), Ciardullo and co-workers (e.g., 1987), Sharov \& Alksnis (1991, 1992, 1997), and Shafter and co-workers (e.g., Shafter \& Irby 2001). More recent efforts include, in particular, the AGAPE project (Ansari et al. 2004), the POINT-AGAPE project (Darnley et al. 2004), and the WeCAPP project (Fliri et al. 2006). The result of this huge effort is a relatively large number of known novae in M31. Recently, PHS07 collected 719 optical novae and nova candidates in M 31 with outbursts before the end of 2005 based on their own research and a search in the literature. This catalogue is regularly updated ${ }^{1}$. PHS07 point out that position information (for some also time of outburst) is poor for many of the early nova detections. The positions could be significantly improved by re-analysing of the original plates.

\footnotetext{
1 available via the Internet at

http://www.mpe.mpg.de/ m31novae/opt/m31
} 


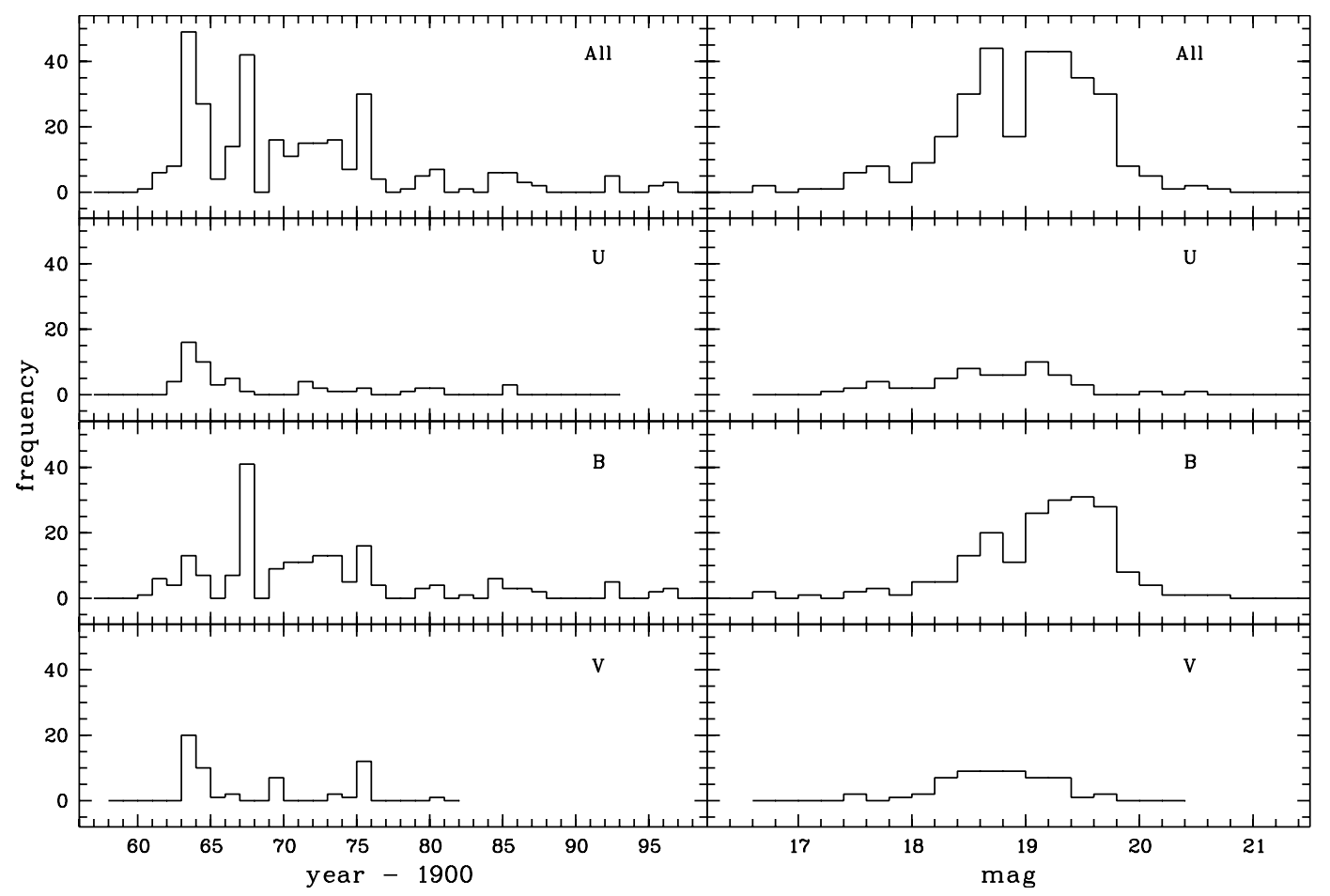

Fig. 1. Left: distribution of epochs of the sample plates for all plates and $U, B, V$ plates, respectively. Right: distribution of limiting magnitude for all sample plates, as well as for $U, B$, and $V$ plates separately. For the definition of limiting magnitude in this work see text and Fig. 2.

Accurate positions - also for historical novae - are important for identifying novae in different wavelength regimes, and especially for secure identification of recurrent novae.

A successful search for optical novae over the whole body of M31 requires a large set of observations that have to be deep enough and have to cover a sufficiently large ( 10 square degrees) field. For such an aim, the plate archives of large Schmidt telescopes provide very useful observational material. The present study is concerned with the M31 plates in the archive of the Tautenburg Schmidt telescope (Sect. 2). Some plates, in particular the oldest ones, have been used already for nova searches by eye (Moffat 1967; Börngen 1968; Meinunger 1975). Here we describe the first automised, systematic nova search in the M31 field on a large and complete selection of digitized Tautenburg Schmidt plates of the M31 field.

Preliminary results of the present study have already been briefly summarised (Henze et al. 2006). The present paper includes a more detailed description of the observational data (Sect. 2), the data reduction (Sect. 3), the calibration (Sect. 4), and the procedures for selecting nova candidates (Sect. 5). The results are presented in Sect. 6 and discussed in Sect. 7. Finally, a summary is given in Sect. 8 .

\section{Observations}

This work is based upon photographic plates taken in the years 1960 to 1996 with the Tautenburg Schmidt telescope (free aperture $1.34 \mathrm{~m}$, focal length $4 \mathrm{~m}$ ). A single Tautenburg plate covers an unvignetted field of $3.3 \times 3.3$ with a plate scale of 51 ". 4 per mm. With 554 plates in total, the M 31 field is the most frequently observed field in the Tautenburg Schmidt archive. We selected a sample of 289 plates in the $U B V$ bands, plus 17 blue-sensitized plates without filter (NFBS). The main selection criterion was the exposure time, which was chosen to be $\geq 10 \mathrm{~min}$ as a basic requirement for a sufficiently deep plate limit.
Table 1. Numbers of plates per colour band.

\begin{tabular}{lllr}
\hline \hline Colour band & Emulsions & Filter & Number \\
\hline$U$ & 103a-O, ZU2, IIa-0 & $U G 2$ & 57 \\
$B$ & 103a-O, ZU2, ZU21, IIa-O, AS & $G G 13$ & 176 \\
$N F B S$ & ZU2, ZU21, AS & no filter & 17 \\
$V$ & 103a-D, ZO1, IIa-D & $G G 11$ & 56 \\
\hline
\end{tabular}

Figure 1 shows the distribution of epochs of the sample plates and Table 1 gives the number of plates per colour band and summarises the emulsions and filters used. Parameters for the individual plates are summarised in Tables 10-19. The Tautenburg colour bands $U B V$ are almost identical to the Johnson system (e.g., van den Bergh 1964; Andruk et al. 1994; Brunzendorf 2001). The NFBS system is close to the B system and, in the context of the present paper, the two systems are considered to be nearly identical.

The distributions of the limiting magnitudes of the selected plates are shown in Fig. 1, where the limiting magnitude is defined here as the peak of the magnitude distribution as shown in Fig. 2 (i.e., the faintest objects detected on a plate are typically $\sim 1$ mag fainter). Note that the limiting magnitude varies significantly across the field due to the bright and spatially variable background from M31. Especially for objects in the bulge, the limiting magnitude is significantly brighter, and inside a certain limiting isophote, the probability detecting stellar objects approaches zero (see Sect. 3 for the object detection). From the statistics of the detected stars from the external calibration catalogue (Sect. 4), we estimate the semi-major axes of these limiting isophotes to $\sim 3^{\prime}$ in $B$ and $\sim 6^{\prime}$ in $V$, respectively. Based on the surface photometry of M 31 given by Walterbos \& Kennicutt (1987), these limits correspond to a limiting surface brightness of $19.80 \mathrm{mag} \operatorname{arcsec}^{-2}(B)$ and $19.73 \mathrm{mag} \operatorname{arcsec}^{-2}(V)$. In the $U$ band, the semi-major axis of the limiting isophote is as small 


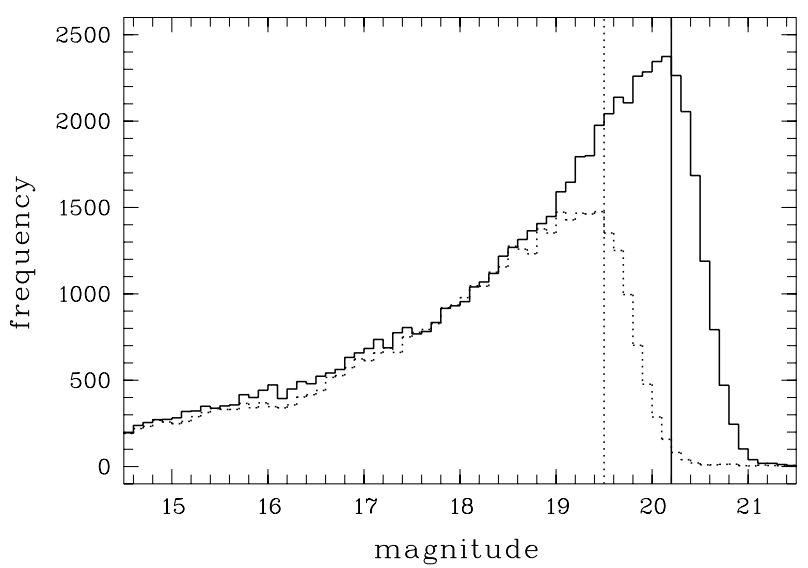

Fig. 2. Distribution of calibrated magnitudes (number per 0.1 mag interval) for all objects detected on photometric reference plates (solid: $B$ plate 8171 , dotted: $U$ plate 1101). Straight vertical lines indicate the limiting magnitudes defined here by the peaks in the distributions $\left(B_{\lim }=20.2, U_{\lim }=19.5\right)$.

as $\sim 1^{\prime}$ where no surface brightness is available from Walterbos $\&$ Kennicutt (1987).

Obviously, these plates constitute valuable observational material suited to searching for bright variables in our neighbour galaxy. We recall that the spectral properties of the plate emulsions, the filters, and the optics have not changed significantly over the years; hence, the plate material is remarkably homogeneous over this long time base. Note, however, that the majority of these plates were not taken as a part of a systematic survey and that the plates thus do not cover exactly the same field.

\section{Data reduction}

All plates were digitized with the Tautenburg Plate Scanner (see Brunzendorf \& Meusinger 1999, for a brief description). The digital images have a pixel size of $10 \mu \mathrm{m} \times 10 \mu \mathrm{m}(0$ ". $5 \times 0$ ". 5$)$ and a resolution depth of 12 bit perpixel. The images were reduced with the source detection software package Source Extractor (SE; Bertin \& Arnouts 1996). The SE creates output tables containing internal $x$-, $y$-positions, internal magnitudes, and additional object parameters. The subsequent data reduction and analysis were done using the ESO MIDAS package.

We used the SE in the Photo-Mode (DETECT_TYPE: PHOTO), which takes the nonlinear response of the photographic emulsions into consideration. Fluxes were measured with a flexible elliptical aperture (SE mode AUTO). Special care was taken to consider the strongly fluctuating background surface brightness. To do so, we configured the SE to model a detailed background image (SE parameters BACK_SIZE $=7$, BACK_FILTERSIZE $=7$ ) using median filters. In an area of BACK_SIZE, the mean and the standard deviation $\sigma$ were computed, and all pixel values exceeding $\pm 3 \sigma$ rejected. This procedure was repeated iteratively until all remaining pixel values lay within $\pm 3 \sigma$ of the current mean value. A background map was built from all areas of BACK_SIZE using the current mean of the area as a value for the background. Additionally, a smoothing median filter was applied to groups of BACK_FILTERSIZE areas to model the final background image. This image was subtracted from the original image and object detection performed on the background-subtracted image. We decided to use a very low detection threshold (SE parameters DETECT_THRESH = 1.3, DETECT_MINAREA = 5) in order to reach a high completeness still at the plate limit. This typically yields $\sim 50000$ detected objects per plate. The big drawback to the high completeness is, of course, a strong contamination by spurious detections that have to be considered carefully in the subsequent data analysis.

To judge the reliability of the detections, we used the SE output parameters FWHM_IMAGE (computed in units of pixel assuming a Gaussian PSF; stars have FWHM 7..15), ELLIPTICITY (stars: $0.05 \ldots 0.1$ ), FLAGS (image reduction errors), and CLASS_STAR. The last parameter is useful for distinguishing between unresolved objects ("stars"; CLASS_STAR close to 1) and extended objects (mainly background galaxies; CLASS_STAR close to 0). This classification is based on a Neural Network. Since the reliability of the CLASS_STAR parameter breaks down for fainter objects (CLASS_STAR distributed randomly between $0 \ldots 1$ ), we computed the additional index NONSTELLAR, which is based solely on the deviation of the measured object profile from the mean profile:

$$
\text { NONSTELLAR }=\frac{r-\tilde{r}}{\sigma_{r}},
$$

where $r$ is the radius of the object, $\tilde{r}$ the median radius of all objects with similar (internal) magnitudes, and $\sigma_{r}$ the standard deviation of the radii of all these objects with $r \leq \tilde{r}$. Objects with NONSTELLAR $>3$ are not considered as stellar.

\section{Calibration}

The astrometric and photometric calibrations follow a similar procedure to previous studies based on digitized Tautenburg Schmidt plates (e.g., Scholz et al. 1997; Brunzendorf \& Meusinger 2001). First, deep reference plates are selected for each filter band and are then calibrated by means of an external catalogue. All other plates are calibrated using the large number of objects from the respective reference plate.

\subsection{Astrometric calibration}

The astrometric calibration of the reference plate (2588) is done in the following steps: (1) selecting an appropriate reference catalogue, (2) matching the SE output table from the plate to the reference catalogue, and (3) transforming the internal plate coordinates into the reference system defined by the catalogue. For the first step, the USNO-B1.0 catalogue (Monet et al. 2003) was used. The catalogue query routine VizieR (Ochsenbein et al. 2000) at the CDS, Strasbourg, yields $\sim 55000$ objects in the field with a position accuracy of $\leq 0$ ". 1 . For these objects rectangular coordinates were computed by VizieR and rescaled to the plate coordinate system.

To match the external catalogue to the catalogue of objects detected on the plate, we used a routine developed at Tautenburg (Brunzendorf 2001), which is based on the MIDAS routine find/pair. First the 100 brightest objects in both tables are matched. In case of success, the coordinate systems are adjusted. This procedure is iteratively repeated with an increasing number of objects (up to $\sim 20000$ matches) and with increased precision. For calibration purposes, we did not use all the matched objects but selected a sample of $\sim 7000$ objects with (a) zero proper motion according to the USNO catalogue and (b) star-like image profile. With those objects of high reliability, we computed 


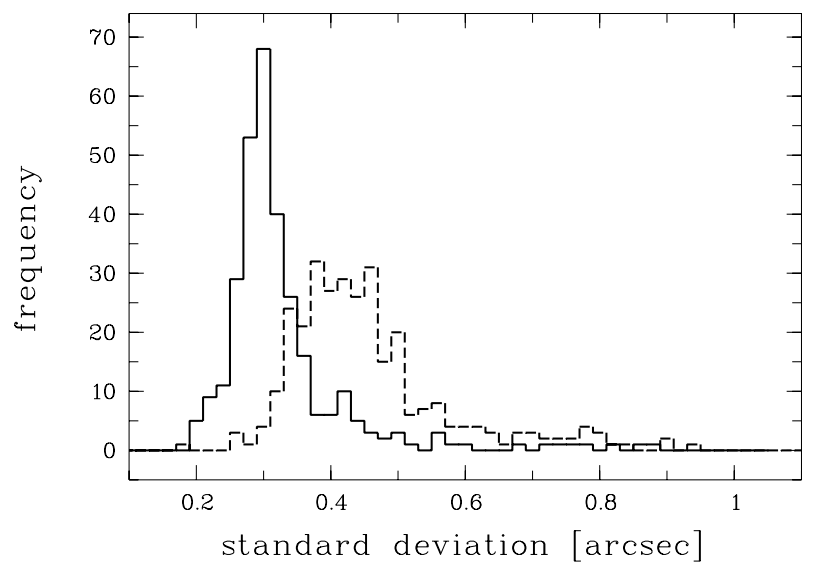

Fig. 3. Distribution of the standard deviations of coordinate differences between all astrometric calibrated plates and the USNO-B1.0 catalogue. Solid curve: objects with $m_{\text {cal }}=16 \ldots 18$ mag; dotted curve: objects with $m_{\text {cal }}>18$ mag.

transformation relations from pixel coordinates to equatorial coordinates using two-dimensional polynomials of third order:

$\alpha=\sum_{i, j=1}^{3} a_{i j} x^{i} y^{j}, \quad \delta=\sum_{i, j=1}^{3} b_{i j} x^{i} y^{j}$.

The calibration of all other plates using the reference plate essentially follows the same method. Figure 3 shows the distribution of astrometric standard errors for all plates. The resulting mean position accuracy is estimated to 0 ".' 3 for objects with calibrated magnitudes (see Sect. 4.2) between 16 and 18 and to 0 ".45 for objects with calibrated magnitudes greater than 18 . The overall astrometric accuracy is estimated to 0 ". 4

\subsection{Photometric calibration}

For the photometric calibration, separate reference plates were used for each of the three filter bands. The external catalogue used here is the M31 part of the Local Group Survey (LGS; Massey et al. 2006). The LGS maps 2.2 square degrees along the major axis of M31 using a mosaic of CCD images. With $U B V$ measurements for 20000 to 30000 stars (uncertainties $\leq 0.01 \mathrm{mag}$ ), the LGS provides an exceptionally large calibration sample. Because the plates have already been astrometrically calibrated in the previous step, the equatorial coordinates could be used for matching the catalogue of objects on the reference plate with the LGS.

We find that the relation between the internal magnitudes and the catalogue magnitudes is not satisfactorily modelled by a single polynomial over the whole magnitude range (calibrated magnitudes $\sim 12 \ldots 21$ ). Therefore, a relation is used consisting of separate regressions for three different magnitude intervals with smooth transitions between these intervals. In this way a good and robust fit is achieved by using combinations of linear and quadratic regressions. Increasing the order of the polynomials, on the other hand, does not produce better results. For each magnitude interval, the calibrated magnitudes $m_{\text {cal }}$ are modelled by

$m_{\mathrm{cal}}=\sum_{i, j}^{2} c_{i, j} m_{\mathrm{int}}^{i} b^{j}$,

where $m_{\text {int }}$ is the internal magnitude of the object and $b$ its background intensity as measured by the SE. The inclusion of the local background as a parameter takes into account that the correct

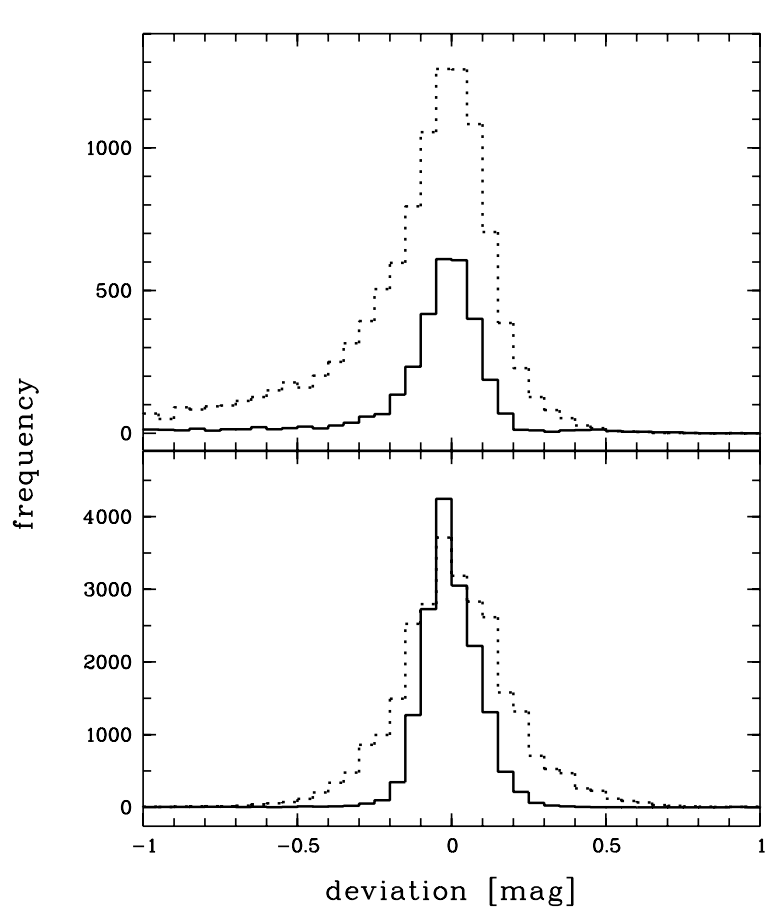

Fig. 4. Top: distribution of the magnitude differences for all objects on the $B$ reference plate 8171 matched with LGS objects. $U$ and $V$ reference plates show similar statistics. Solid line: $m_{\text {cal }}<18$ mag; dotted line: $m_{\text {cal }}>18$ mag. Bottom: the same for typical magnitude differences for photometric calibrated plates shown with matched objects of plates 8171 and 8089.

background subtraction is always difficult for a non-linear detector, especially when the background is bright and has a high spatial frequency. We find that the photometric accuracy is indeed significantly improved in this way $(0.1 \ldots 0.2$ mag better). Furthermore, we confirm earlier results (e.g., Brunzendorf 2001) that including colour terms does not yield a substantial improvement of the fit over including the background.

For the calibration of all the other plates, the field was subdivided into two different areas that are complementary and separated by an ellipse approximating the contours of the outer spiral arms of M 31. The region inside this ellipse contains the area with the strong and spatially varying background of the disk. The outer region is characterised by a much fainter and relatively homogeneous background light distribution. This separate calibration approach yields a further improvement in the photometric accuracy. The calibration itself is done in the same way as described above for the reference plates. Both procedures work semi-interactively, where every single plate needs an individual setting of the magnitude intervals and of the order of polynomials used for the regression. Figure 4 illustrates the results of the photometric calibration.

\section{Data analysis}

\subsection{Creation of light curves}

To create light curves for all detected objects, it is first necessary to match the object catalogues from the different plates. Previous searches for variable sources on digitized Schmidt plates (Scholz et al. 1997; Brunzendorf \& Meusinger 2001) started from a basic object sample defined as the sample of all objects detected on a minimum number of plates. However, such an approach cannot 
be applied here because the time span of the observability of extragalactical novae can be as short as a few days. Also, the irregular sampling of the observations has to be taken into account. Thus it is possible that novae are detected on only two plates of similar epochs. To get light curves of all objects detected on at least two plates, every plate has to be correlated with every other plate. This was done here for every colour band separately. Of course a nova can also be detected on only one plate of a colour band. We define the detection in this case not as a light curve and discuss these objects in Sect. 5.3.

The correlation procedure works as follows. The field actually considered is the field of the astrometric reference plate. For every other plate, only the overlap region with the reference plate field was used for correlation. The reference field was subdivided into segments with a size of $0^{\circ} 1 \times 0^{\circ} .1$. For each of these sub-areas, the coordinates of all objects in the first plate table were matched with the coordinates of all other objects in the respective part of the field of all other plates. Since the typical position accuracy for a single plate in comparison to the reference catalogue is $\sim 0$." 4 (see Sect. 4.1 and Fig. 3), the typical position error for the comparison of two plates is $\sigma \sim \sqrt{2} \times 0$ ". 4 . Hence we used an identification radius of $2^{\prime \prime}$ corresponding to $\sim 3.5 \sigma$. This correlation procedure was repeated iteratively for all objects of the remaining plates that could not be identified in previous steps. By this means, the highest completeness could be achieved since every object on every plate was, in principle, compared with all objects on all other plates. The segmenting of the field was done with the large number of plates and the huge number of $\sim 45000$ detections for every plate in mind, in order to speed up the correlation procedure. Matching problems at borders of segments could be solved using overlap regions and selection criteria avoiding double matching.

For each colour band, the correlation procedure produced tables (a) for all objects detected on at least two plates ("multidetection objects") and (b) for all objects detected on only one plate ("single-detection objects"). Both tables contain object coordinates and magnitudes for every plate the object was detected on. For multi-detection objects, the coordinates on the reference plate were used, if existing, or the coordinates on the first plate the object was detected on. Knowing the associated Julian Date (JD) for every plate, we were able to create light curves for every object. The analysis of the multi-detection light curves is described in Sect. 5.2. Single-detection objects need to be treated differently and are discussed in Sect. 5.3.

\subsection{Selection of nova candidates from light curves}

In a first step, the non-stellar objects were removed from the tables by a routine based on the geometric parameters (see Sect. 3). As criteria for a star-like appearance we used: CLASS_STAR $\geq 0.33$, NONSTELLAR $\leq 6$, FWHM_IMAGE $\leq 18$ (to remove extended objects and very bright foreground stars), ELLIPTICITY $\leq 0.15$ (to remove background galaxies and the most obvious plate defects), and FLAGS $\leq 3$ (to remove serious reduction errors). Every object not satisfying at least one of these constraints was removed. These criteria are quite weak and each of them defines a lower limit. The reason for using weak constraints is to avoid a strong reduction of the sample at faint magnitudes where all these parameters are uncertain. Although this preselection only removes the obviously non-star like objects, the total number of objects is reduced by up to $30 \%$ (see Table 2). Note that in this step overlapping
Table 2. Total number of objects in multi-detection tables.

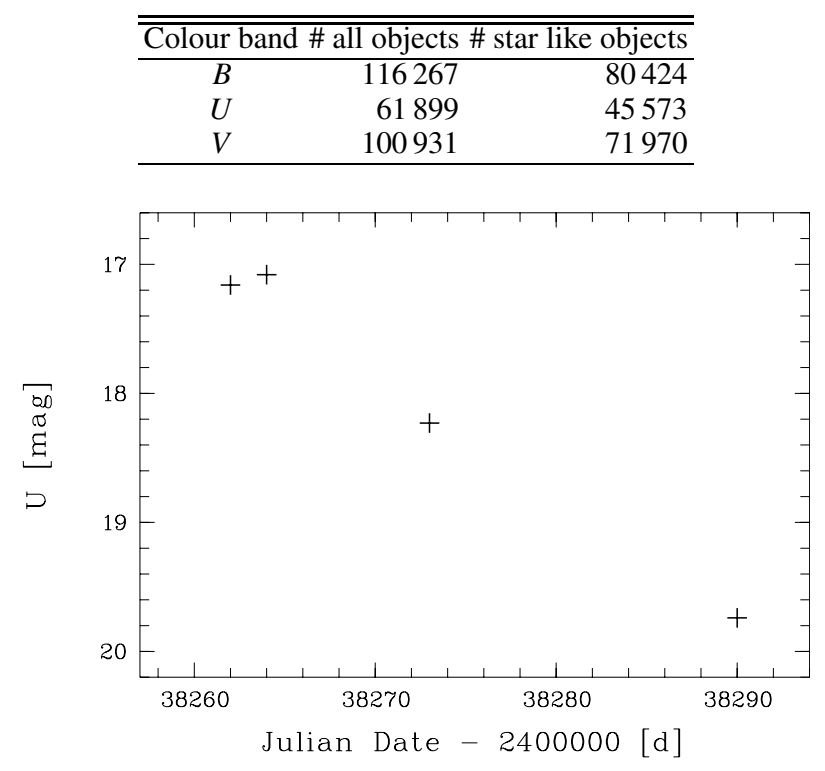

Fig. 5. Light curve of nova candidate $4=$ Nova Moffat $2=$ M31N 196308a. The light curve parameters are $j d d i f f=28 \mathrm{~d}$, limitdiff $=2.0 \mathrm{mag}$, and peakdiff $=8.1$. Detection images of this object are shown in Fig. 6 below.

stellar images are also rejected, which could not be deblended correctly by the Source Extractor.

For the remaining objects, we computed light curve parameters specifically designed to select nova candidates. These parameters are (a) the time lag between the first and the last detections of the object (jddiff), (b) the number of plates (nop) the object was detected on, (c) the magnitude difference of the limiting magnitude for the particular plate that the light curve peak was detected on to this peak magnitude (limitdiff), and (d) the probability that this peak is not due to a random photometric fluctuation (peakdiff). The last parameter is computed as follows: the peak measurement is removed from the light curve and the mean magnitude is computed from this modified light curve. Peakdiff now gives the difference between this modified mean and the actual peak value in terms of the standard deviation of the respective plate in the respective magnitude interval. The standard deviation is computed in the following way: the sample of multi-detection objects is binned into 0.5 mag intervals according to their magnitude on the reference plate. Then the differences between the object magnitudes on this plate and on the reference plate are computed for each calibrated plate. The distribution of these differences is represented by a Gaussian (see Fig. 4 for an example) and the standard deviation can be used to make individual estimates of the uncertainties on the photometric calibration. The mean standard deviation over all plates and all magnitude intervals is $\sim 0.2 \ldots 0.3 \mathrm{mag}$. We decided to use low values for peakdiff in order to be able to discover nova light curves that have not been detected close to the maximum. This, of course, results in a large number of objects having to be checked carefully.

To interpret these parameters we analysed the data for the novae detected by Moffat (1967) and Börngen (1968) on Tautenburg plates. A sample of 20 typical nova light curves was constructed (Fig. 5 for illustration), and their parameters were used to define the region of the parameter space where novae should be searched. Not all of the known novae were included, 


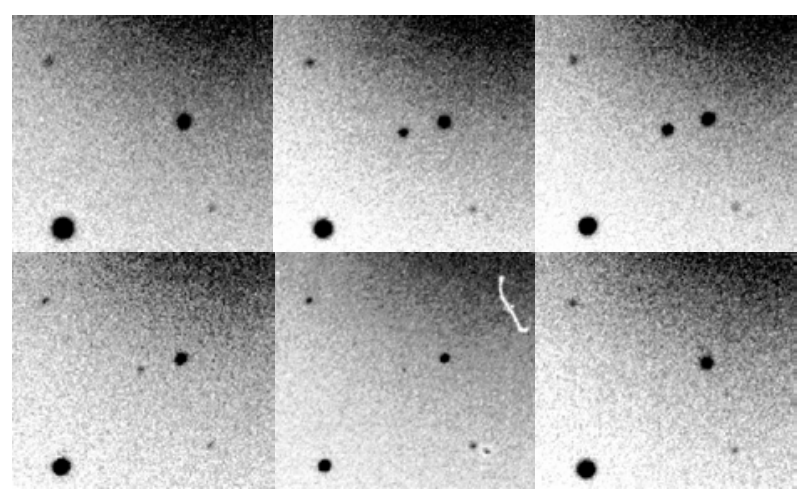

Fig. 6. Images of nova candidate 4 on Tautenburg $U$ plates. Frames are sorted chronologically from left to right and from top to bottom. Frame size is $\sim 2^{\prime} \times 2^{\prime}$, and north is up and east is left. The object appears in the centre of the four middle frames. The first and the last frames show plates 732 and 1260 that surround the nova detections (see also Tables 10-19). The resulting light curve is shown in Fig. 5 above.

and the remaining objects were used to check the approach. We consider four different cases in detail:

1) nop $>2$ : the analysis of these light curves does not require the parameter limitdiff, which would remove too many faint objects;

2) $n o p=2$ : the parameter peakdiff is not useful here so we use instead limitdiff to remove spurious objects. In fact the actual values of limitdiff are quite low and lead to $\sim 100$ selected objects where nova candidates constitute only a small percentage. The whole sample (without exclusion of the faintest objects) yields several hundred objects in which noise detections and plate faults are the vast majority;

3) recurr: here we search for recurrent novae defined by light curves consisting of two parts separated by more than 360 days where at least one part satisfies the selection criteria for case 1 (i.e., $n o p \geq 2$ ).

4) recurr 2: as case 3 but for nop $=2$.

The selection criteria are similar for the three colour bands, but the values of the parameters were individually configured for each band. The final selection parameters are summarised in Table 3. The last two columns give the numbers of selected objects and of final nova candidates, respectively. Note that the criteria for recurrent novae are applied to light curves consisting of two parts separated by more than 360 days.

Two types of objects were automatically rejected by the selection procedure: (1) objects from those parts of the field that are covered by only a small number of plates with small epoch differences and (2) nova candidates obviously mimicked by high-proper motion foreground stars (where a star has moved out of the identification radius over the long time base of our plate material). All other objects satisfying the light curve selection criteria are regarded as "selected objects", and their numbers are given in Table 3 .

In a next step, the light curves of all selected objects are checked individually. In particular, each selected object is correlated with the object lists from the other two colour bands. Several objects selected in one band were removed because the light curve in another band clearly indicates that the object is not a nova. On the other hand, the independent selection of the same object in two or even three colour bands is a very strong argument for a nova candidate. Each object from the final sample of selections was inspected by eye on the digitized plates, mainly to directly check the stellar appearance by comparing
Table 3. Nova selection criteria for the different colour bands.

\begin{tabular}{cllrr}
\hline \hline $\begin{array}{c}\text { Colour } \\
\text { band }\end{array}$ & Case & Parameters & \#sel & \#cand \\
\hline$U$ & nop $>2$ & jddiff $\leq 50$, peakdiff $\geq 1$ & 28 & 11 \\
& nop $=2$ & jddiff $\leq 25$, limitdiff $\geq 0$ & 139 & 10 \\
& recurr & jddiff $\leq 50$, peakdiff $\geq 1$ & 43 & 0 \\
& recurr 2 & jddiff $\leq 25$, limitdiff $\geq 0$ & 139 & 1 \\
$B$ & nop $>2$ & jddiff $\leq 70$, peakdiff $\geq 0.5$ & 119 & 20 \\
& nop $=2$ & jddiff $\leq 5$, limitdiff $\geq 0$ & 107 & 15 \\
& recurr & jddiff $\leq 70$, peakdiff $\geq 0.5$ & 227 & 0 \\
& recurr 2 & jddiff $\leq 5$, limitdiff $\geq 0$ & 77 & 0 \\
$V$ & nop $>2$ & jddiff $\leq 50$, peakdiff $\geq 0.5$ & 70 & 2 \\
& nop $=2$ & jddiff $\leq 20$, limitdiff $\geq 0.5$ & 69 & 5 \\
& recurr & jddiff $\leq 50$, peakdiff $\geq 0.5$ & 445 & 0 \\
& recurr 2 & jddiff $\leq 20$, limitdiff $\geq 0.5$ & 122 & 0 \\
\hline
\end{tabular}

Table 4. Number of all single-detection objects (\#normal priority) and of objects in the high-priority sample, respectively (last column).

\begin{tabular}{crrr}
\hline \hline Colour band & Plates & \#normal priority & \#high priority \\
\hline$U$ & 57 & 253240 & 294 \\
$B$ & 193 & 582211 & 573 \\
$V$ & 56 & 255058 & 182 \\
\hline
\end{tabular}

its image structure with the nearby stars of similar magnitude. Furthermore, we checked via the Aladin Sky Atlas at the CDS, Strasbourg, if the objects are visible on more than one DSS POSS II plate (of course not expected for a nova) and if the objects already have entries in the databases of SIMBAD and NED. All objects passing all tests successfully constitute our sample of nova candidates. The corresponding numbers from the three bands are listed in Table 3.

\subsection{Selection of nova candidates among the single detections}

The number of single-detection objects is much greater than the number of multi-detection objects (see Tables 2 and 4). Reasons for this are (a) a large number of noise detections due to the low detection thresholds, (b) various "plate defects", and (c) the fact that several of the older plates had been broken in the past. These plates have been repaired, which results, however, in a significant loss of astrometric accuracy and consequently in matching problems and wrong identifications in the context of creating the light curves.

To select objects of high reliability, the measurements on heavily damaged plates were completely removed from the analysis of the single detections. For the remaining plates we considered only objects brighter than 1 mag below the plate limit and applied the following selection criteria based on the image structure: CLASS_STAR $\geq 0.8$, ELLIPTICITY $\leq 0.1$, FLAGS $\leq 3$, FWHM_IMAGE $\leq 15$, and NONSTELLAR $\leq 3$. The objects satisfying all these criteria constitute the sample of high priority in contrast to the whole sample of single detections that have only normal priority. The constraints are much stronger here than for the multi-detection objects. The high-priority sample therefore contains only about $0.1 \%$ of all single detections (Table 4 ). However, even this high-priority sample is still heavily contaminated by plate defects that can perfectly mimic star-like objects. Although there are elaborate methods known to distinguish between plate defects and stars (e.g., Greiner et al. 1990), such techniques are too expensive to be applied to the several hundred objects in our study. A single detection therefore is not enough 
Table 5. Correlations of single detections.

\begin{tabular}{llr}
\hline \hline First table & Second table & \#cand \\
\hline single high priority & multi & 16 \\
single high priority & single high priority & 1 \\
single normal priority & PHS07 & 24 \\
\hline
\end{tabular}

for classifying an object as a nova candidate. In the context of the present work, the only way to restrict the large number of single detections to a sizable candidate sample is to correlate the table of single-detection objects with other tables in order to get additional information on these objects. Note that the terms multi-detection object and single-detection object were defined in the context of the light curve analysis. Since light curves were constructed for every colour band independently, it is possible that e.g. two single detections from different colour bands show a position coincidence. Therefore, we defined identification criteria for nova candidates by referring to the position difference (dist) and the time lag (diff) of two records in different tables to be identified as the same object. We matched the high-priority samples of each colour band with the tables of multi detections or high priority single detections in the other colour bands. The identification criterion in these cases is dist $\leq 2^{\prime \prime} \&|\mathrm{diff}| \leq 50 \mathrm{~d}$. Additionally, we matched the normal priority single detections with the table of the already known novae to find out which novae we can confirm and to search for recurrent novae. Here we used (a) dist $\leq 10^{\prime \prime} \& \mid$ diff $\mid \leq 100$ d for identifying novae to confirm and (b) dist $\leq 10^{\prime \prime} \& \mid$ diff $\mid>100 d$ for identifying recurrent nova candidates. Table 5 shows the results of these correlations. The first two columns give the matched tables and the third column gives the number of nova candidates discovered or confirmed using correlations.

Furthermore, we matched the normal priority single detections in a given colour band with the normal priority single detections in the other colour band. Because of the high number of objects, we used stricter identification criteria (dist $\leq 1^{\prime \prime} \&$ $\mid$ diff $\mid \leq 50 \mathrm{~d}$ ). We did not find any additional nova candidates with these selection criteria.

\section{Results}

Using the criteria detailed in the previous section we found in total 55 nova candidates based solely on data from the Tautenburg plates; details are given in Tables 6-8. Table 6 contains all objects that were previously discovered on Tautenburg Schmidt plates by Moffat (1967), Börngen (1968), and Meinunger (1975) and that were confirmed here. Table 7 contains all selected nova candidates that match already known novae from the literature that have not been measured before on Tautenburg plates. Especially for the candidates in Table 7, the independent detection and classification as nova candidates makes them very likely to actually be novae. Table 8 presents all nova candidates that were newly detected in this study. For nova candidates in Table 8, no counterparts were found in the nova catalogue (PHS07) having a position difference of $\leq 60^{\prime \prime}$ and a time lag of less than 100 days.

Furthermore, we confirm 29 known novae mostly found by analysing matches of the single-detection objects with PHSO7 (Table 9). Some of these objects have more than one single detection, which could not be correlated, however, because one or more of these detections were made on broken plates having large astrometric errors. Hence these objects were not found independently using the analysis of the light curves, but they do confirm already known nova candidates. For all of these objects, we present significantly improved positions. A second confirmation exists for each of these objects, either a second single detection, a faint but not detected object found by eye on one of our sample plates, an object found by eye on a plate not included in the original plate sample (e.g. an $R$ plate), or an entry in PHS07. To judge the possibility that bright nova candidates appear only as one single detection, we analysed the high-priority sample of the $U$ band single detections (see Table 4) and checked the digitized images of the objects. All but two objects were characterised as plate defects due to their geometry in comparison to nearby stars or because the objects cannot be found on subsequent neighbour plates with small epoch differences to the detection plate. We found the remaining two objects to be nova candidates using correlations described in Sect. 5.3. Therefore, we assume that no bright nova candidate was missed that only appears as a single detection. Some nova candidates in Tables 69 (indicated by $(*)$ ) show specific features in their identification. We discuss them individually in Sect. 7.2.

We present finding charts for all 84 novae in Figs. 8(a)-14(1). All these charts have a size of $4^{\prime} \times 4^{\prime}$ and are oriented north up and east left. Light curve data points of 82 nova candidates detected on the plates (see Sect. 7.2 for the remaining two) are given in Tables 10-19. Figure 7 shows the spatial distribution of the novae over the disk of M31.

We found no light curve unambiguously showing a recurrent nova in our data base. One candidate was selected using the model for recurrent novae shown in Table 3, but the subsequent visual inspection revealed an incorrect identification with a nearby star on a broken plate. However, one of our newly found novae correlates with a known nova and is therefore likely to be a recurrent nova. We also discuss this object in Sect. 7.2.

The observed maximum brightness of all nova candidates is distributed as follows: 3 novae are brighter than 16 mag, 6 novae range between 16 and $17 \mathrm{mag}, 29$ novae range between 17 and $18 \mathrm{mag}$, and 44 novae are fainter than $18 \mathrm{mag}$. For 7 novae, we estimated $t_{2}$, i.e. the time (in days) taken for the luminosity to fall 2 mag below the (observed) maximum. We give the speed class for these objects according to Payne-Gaposchkin (1957) and discuss these objects in Sect. 7.2. Most of the previously known novae were detected on Tautenburg plates after the already known outburst. However, note that 9 novae were detected $\leq 5$ days before the previously known outburst and 3 novae even earlier (up to 84 days before). These 3 objects are also discussed in Sect. 7.2.

\section{Discussion of individual nova candidates}

Before we discuss individual nova candidates, we want to emphasise that we searched explicitly for objects that were visible only on a short time span. Consequently this means that the seldom novae in globular clusters (like in Quimby et al. 2007) are not recognised by this procedure if those clusters are bright enough to be detected. A search for such novae could be made in the context of an analysis of long-term variable objects, which we plan to conduct in the future.

In addition, we want to point out that the results presented in the present paper contain a few modifications compared to the preliminary results published by Henze et al. (2006) where 19 new historical nova candidates in M 31 have been announced. Meanwhile, we identified one of these objects (nova 32, discussed below) with an already known nova and found four additional new nova candidates (novae 35, 36, 42, and 43). 
Table 6. Basic data for the previously known Tautenburg nova candidates.

\begin{tabular}{cccclrrrr}
\hline \hline $\mathrm{Nr}^{a}$. & $\alpha\left[^{\circ}\right]^{b}$ & $\delta\left[^{\circ}\right]^{b}$ & $\mathrm{Mag}^{c}$ & Name $^{d}$ & $d\left[^{\prime \prime}\right]^{e}$ & $t[\mathrm{~d}]^{e}$ & ${\mathrm{JD}[\mathrm{d}]^{f}}^{\text {Year }^{g}}$ \\
\hline 1 & 9.46035 & 41.35378 & $19.3(\mathrm{~B})$ & M31N1962-08b ([M75],S10754) & 2.0 & 30 & 2437913 & 1962 \\
2 & 10.41910 & 41.05943 & $17.2(\mathrm{U})$ & M31N1962-11a ([B68],N05) & 5.4 & 0 & 2437988 & 1962 \\
3 & 10.14533 & 40.73047 & $17.8(\mathrm{U})$ & M31N1962-09b ([B68],N03) & 9.5 & 75 & 2437989 & 1962 \\
$4\left(^{*}\right)$ & 10.69604 & 41.22367 & $17.1(\mathrm{U})$ & M31N1963-08a ([M67],N2) & 0.3 & 0 & 2438264 & 1963 \\
$5\left(^{*}\right)$ & 10.88359 & 41.35630 & $15.4(\mathrm{U})$ & M31N1963-09b ([M67],N1,R047) & 4.6 & -2 & 2438284 & 1963 \\
6 & 11.11041 & 41.65485 & $19.1(\mathrm{U})$ & M31N1963-09a ([M67],N3) & 19.5 & 1 & 2438284 & 1963 \\
$7\left(^{*}\right)$ & 10.22257 & 40.99426 & $17.4(\mathrm{U})$ & M31N1963-10b ([B68],N08) & 2.4 & 12 & 2438322 & 1963 \\
8 & 10.78286 & 41.37024 & $17.6(\mathrm{U})$ & M31N1963-10e ([B68],N09) & 1.2 & 3 & 2438328 & 1963 \\
9 & 10.39708 & 40.98540 & $18.0(\mathrm{~V})$ & M31N1963-12a ([B68],N11) & 1.7 & 6 & 2438373 & 1963 \\
10 & 10.57277 & 41.12444 & $17.1(\mathrm{U})$ & M31N1963-10d ([B68],N10) & 1.0 & 51 & 2438373 & 1963 \\
11 & 11.01297 & 41.26195 & $17.6(\mathrm{U})$ & M31N1964-09a ([B68],N13) & 0.9 & 23 & 2438675 & 1964 \\
12 & 10.69894 & 41.28864 & $17.7(\mathrm{U})$ & M31N1964-10a ([B68],N14) & 2.3 & 2 & 2438672 & 1964 \\
13 & 10.72437 & 41.36870 & $17.6(\mathrm{U})$ & M31N1965-06a ([B68],N15) & 2.2 & 68 & 2439007 & 1965 \\
14 & 10.64249 & 41.16618 & $17.2(\mathrm{U})$ & M31N1966-09b ([B68],N20) & 0.4 & 11 & 2439389 & 1966 \\
15 & 10.48746 & 41.13616 & $18.7(\mathrm{~B})$ & M31N1967-08a ([B68],N22) & 1.3 & 29 & 2439735 & 1967 \\
16 & 10.50658 & 41.06845 & $19.0(\mathrm{~B})$ & M31N1967-09a ([B68],N24) & 3.2 & 28 & 2439767 & 1967 \\
$17(*)$ & 10.90417 & 41.31848 & $16.9(\mathrm{~B})$ & M31N1967-10b ([M75],S10731) & 13.4 & 0 & 2439789 & 1967 \\
$18(*)$ & 11.08395 & 41.56903 & $15.2(\mathrm{~B})$ & M31N1967-10c ([B68],N26) & 2.4 & 0 & 2439793 & 1967 \\
19 & 10.82215 & 41.32761 & $17.3(\mathrm{~B})$ & M31N1967-10a ([B68],N25) & 1.6 & 18 & 2439793 & 1967 \\
20 & 8.68975 & 40.84419 & $18.6(\mathrm{~B})$ & M31N1967-10d ([M75],S10752) & 30.5 & -2 & 2439798 & 1967 \\
21 & 9.86471 & 40.73456 & $19.5(\mathrm{~B})$ & M31N1967-08b ([B68],N23) & 3.5 & 67 & 2439798 & 1967 \\
\hline
\end{tabular}

Notes: $\quad{ }^{a}$ Identification number; $(*)$ indicates objects discussed in Sect. 7.2.

${ }^{b}$ Right ascension and declination are given in J2000.0.

${ }^{c}$ Magnitude and colour band of the detected maximum.

${ }^{d}$ Names of the objects following the naming scheme of PHS07; in parentheses we give the original names where M67 = Moffat (1967), B68 = Börngen (1968), M75 = Meinunger (1975), R = Rosino (1973).

${ }^{e}$ Position difference $d$ and time lag $t$ between the present study and the previously published data; the time lag is computed as $t=j d_{\text {here }}-j d_{\text {previous }}$, i.e. negative values mean an earlier detection on our plates than in previous works.

${ }^{f}$ Julian Date of maximum luminosity on Tautenburg plates; for the first detection on Tautenburg plates see Tables 10-19

${ }^{g}$ Year of the outburst.

Table 7. As for Table 6 but for the previously known nova candidates which have not been reported before on Tautenburg plates.

\begin{tabular}{lccclrrrc}
\hline \hline Nr. & $\alpha\left[^{\circ}\right]$ & $\delta\left[^{\circ}\right]$ & Mag & Name $^{a}$ & $d\left[^{\prime \prime}\right]$ & $t[\mathrm{~d}]$ & JD [d] & Year \\
\hline 22 & 10.67795 & 41.23536 & $18.2(\mathrm{~B})$ & M31N1969-10a (R086) & 0.6 & 2 & 2440508 & 1969 \\
23 & 10.43157 & 41.05191 & $18.5(\mathrm{~B})$ & M31N1972-11a (R097) & 6.1 & -2 & 2441621 & 1972 \\
$24(*)$ & 10.20390 & 40.83336 & $16.1(\mathrm{~B})$ & M31N1973-10b (ShA1,N15) & 13.5 & -3 & 2441980 & 1973 \\
25 & 10.66535 & 41.08436 & $18.9(\mathrm{~B})$ & M31N1973-10a (R102) & 0.2 & 6 & 2441984 & 1973 \\
26 & 10.50494 & 41.19690 & $18.3(\mathrm{~B})$ & M31N1974-08a (R105) & 1.1 & 4 & 2442276 & 1974 \\
27 & 10.76844 & 41.22392 & $19.1(\mathrm{~B})$ & M31N1975-10a (R110) & 1.4 & 19 & 2442718 & 1975 \\
$28(*)$ & 10.88516 & 41.33082 & $18.2(\mathrm{U})$ & M31N1985-09g (R139) & 0.2 & -21 & 2446302 & 1985 \\
29 & 10.38892 & 41.03891 & $17.4(\mathrm{U})$ & M31N1985-09h (ShAl,N18) & 23.5 & -5 & 2446322 & 1985 \\
30 & 10.60396 & 41.30883 & $17.9(\mathrm{~B})$ & M31N1987-08a (ShA1,N32) & 1.9 & 4 & 2447039 & 1987 \\
$31\left(^{*}\right)$ & 10.47412 & 41.12277 & $18.0(\mathrm{~B})$ & M31N1992-12b ([S120011],N1992-01) & 0.6 & -84 & 2448893 & 1992 \\
$32\left(^{*}\right)$ & 10.76761 & 40.40784 & $17.3(\mathrm{~B})$ & M31N1996-08d (ShAl,N48) & 46.1 & 2 & 2450316 & 1996 \\
33 & 10.58225 & 41.23554 & $16.4(\mathrm{~B})$ & M31N1996-08g (ShAl,N49) & 1.2 & 0 & 2450317 & 1996 \\
\hline
\end{tabular}

Note: ${ }^{a}$ Names in parentheses refer to $\mathrm{R}=$ Rosino $(1964,1973,1989), \mathrm{ShAl}=$ Sharov \& Alksnis $(1991,1992,1997)$, and SI2001 = Shafter \& Irby (2001).

\subsection{Rejected novae}

We have re-investigated all nova candidates previously found on Tautenburg plates in considerable detail. Most of them were confirmed as nova candidates and are listed in Tables 6 or 9. However, for two objects we found that they have very likely been misclassified as novae, whatever they are instead. These objects will be discussed briefly below.

\section{Nova M31N 1966-10a = Nova Börngen 21}

This nova candidate is described by Börngen (1968) as "nova like, weakly confirmed" and was detected on only three plates.
We find that there is a very faint object visible on all of these three plates; however, we noticed position differences of up to 4 ". 3 between the three objects and therefore do not consider this object as a nova. (There is also no pattern recognisable in this position's differences that can be interpreted as caused by a highproper motion star.)

\section{Nova M31N 1967-08c = Nova Meinunger S10753}

This object was classified as a nova by Meinunger (1975) because of a significant ( $\geq 1 \mathrm{mag})$ outburst that is confirmed in our data. However, there is a very faint object visible at that position on many other plates of different epochs. Moreover, 
Table 8. As for Tables 6 and 7 but for the new nova candidates from the present study.

\begin{tabular}{lccclcc}
\hline \hline Nr. & $\alpha\left[^{\circ}\right]$ & $\delta\left[^{\circ}\right]$ & Mag & Name & JD [d] & Year \\
\hline 34 & 11.90708 & 41.75834 & $19.5(\mathrm{U})$ & M31N1962-09a & 2437911 & 1962 \\
35 & 10.70393 & 41.30974 & $17.7(\mathrm{U})$ & M31N1963-08b & 2438258 & 1963 \\
36 & 11.45452 & 41.89021 & $19.1(\mathrm{U})$ & M31N1963-08c & 2438262 & 1963 \\
37 & 10.01956 & 40.62433 & $18.4(\mathrm{~V})$ & M31N1963-12b & 2438373 & 1963 \\
38 & 11.43947 & 41.75058 & $17.5(\mathrm{U})$ & M31N1966-10b & 2439417 & 1966 \\
39 & 9.33152 & 40.52856 & $18.8(\mathrm{~B})$ & M31N1970-11a & 2440917 & 1970 \\
$40\left(^{*}\right)$ & 10.38148 & 40.87432 & $16.7(\mathrm{~B})$ & M31N1972-01b & 2441328 & 1972 \\
41 & 9.81823 & 40.52356 & $18.8(\mathrm{~B})$ & M31N1972-12b & 2441680 & 1972 \\
42 & 10.48303 & 41.18581 & $15.6(\mathrm{~B})$ & M31N1975-02a & 2442455 & 1975 \\
$43(*)$ & 10.68148 & 41.19253 & $18.5(\mathrm{~B})$ & M31N1975-09a & 2442665 & 1975 \\
$44(*)$ & 10.36907 & 40.88704 & $18.6(\mathrm{~V})$ & M31N1975-11a & 2442741 & 1975 \\
45 & 10.44820 & 40.95410 & $18.6(\mathrm{~B})$ & M31N1975-12b & 2442775 & 1975 \\
46 & 11.47816 & 40.92837 & $19.0(\mathrm{~B})$ & M31N1979-11a & 2444194 & 1979 \\
47 & 10.47874 & 41.01253 & $18.0(\mathrm{U})$ & M31N1980-09b & 2444490 & 1980 \\
48 & 10.14823 & 41.32396 & $17.6(\mathrm{U})$ & M31N1980-09a & 2444490 & 1980 \\
49 & 10.74837 & 41.28688 & $18.0(\mathrm{U})$ & M31N1980-09c & 2444490 & 1980 \\
50 & 11.08057 & 41.60674 & $19.3(\mathrm{~B})$ & M31N1984-08a & 2445940 & 1984 \\
51 & 11.51205 & 41.73243 & $18.6(\mathrm{U})$ & M31N1985-08a & 2446299 & 1985 \\
52 & 11.58009 & 41.97954 & $18.5(\mathrm{U})$ & M31N1985-08b & 2446299 & 1985 \\
53 & 12.51510 & 41.42756 & $18.8(\mathrm{U})$ & M31N1985-08c & 2446299 & 1985 \\
54 & 10.43303 & 41.07269 & $16.5(\mathrm{~B})$ & M31N1992-09a & 2448893 & 1992 \\
55 & 11.54533 & 41.61147 & $19.5(\mathrm{~B})$ & M31N1996-08e & 2450317 & 1996 \\
\hline
\end{tabular}

Table 9. As for Tables 6 and 7 but for the confirmed nova candidates from the present study.

\begin{tabular}{|c|c|c|c|c|c|c|c|c|}
\hline Nr. & $\alpha\left[^{\circ}\right]$ & $\delta\left[^{\circ}\right]$ & Mag & Name $^{a}$ & $d\left[{ }^{\prime \prime}\right]$ & $t$ [d] & $\mathrm{JD}[\mathrm{d}]$ & Year \\
\hline 56 & 10.70850 & 41.47538 & $17.1(\mathrm{~B})$ & M31N1960-11a ([B68],N01) & 0.9 & 8 & 2437261 & 1960 \\
\hline $57(*)$ & 10.63423 & 40.87034 & $18.9(\mathrm{U})$ & M31N1962-08a ([M75],S10730) & 2.3 & 27 & 2437911 & 1962 \\
\hline $58(*)$ & 10.86169 & 41.25254 & $17.8(\mathrm{U})$ & M31N1962-07a ([B68],N02) & 0.5 & 41 & 2437911 & 1962 \\
\hline 59 & 10.61778 & 41.36867 & 17.5 (B) & M31N1962-09d ([B68],N04) & 1.0 & 30 & 2437961 & 1962 \\
\hline 60 & 10.66868 & 41.22996 & $18.7(\mathrm{U})$ & M31N1962-10a ([B68],N06) & 0.9 & 28 & 2437989 & 1962 \\
\hline 61 & 10.74055 & 41.13677 & $18.0(\mathrm{U})$ & M31N1963-09c (R048(079)) & 0.5 & 2 & 2438290 & 1963 \\
\hline $62(*)$ & 10.94180 & 41.64252 & - & M31N1963-10a ([B68],N07) & 1.0 & 0 & 2438311 & 1963 \\
\hline 63 & 11.01505 & 41.43409 & 20.0 (B) & M31N1963-10c ([M75],S10733) & 3.3 & 4 & 2438319 & 1963 \\
\hline $64(*)$ & 10.65329 & 41.36931 & - & M31N1964-08b ([B68],N12) & 2.3 & 7 & 2438621 & 1964 \\
\hline 65 & 12.43549 & 41.57251 & $18.9(\mathrm{~V})$ & M31N1964-09b ([M75],S10755) & 18.5 & 2 & 2438642 & 1964 \\
\hline 66 & 10.30955 & 40.72338 & $18.9(\mathrm{U})$ & M31N1965-09c ([B68],N16) & 2.7 & -1 & 2439025 & 1965 \\
\hline 67 & 11.05491 & 41.78507 & $16.5(\mathrm{~V})$ & M31N1965-11c ([B68],N17) & 4.6 & 0 & 2439088 & 1965 \\
\hline $68(*)$ & 10.60744 & 41.22068 & $19.5(\mathrm{U})$ & M31N1966-09c ([B68],N18) & 2.0 & 9 & 2439389 & 1966 \\
\hline 69 & 10.09839 & 40.40145 & $17.5(\mathrm{U})$ & M31N1966-09f ([B68],N19) & 3.8 & 0 & 2439390 & 1966 \\
\hline 70 & 11.13049 & 41.41552 & $19.4(\mathrm{U})$ & M31N1966-09d ([M75],S10734) & 2.7 & 2 & 2439390 & 1966 \\
\hline 71 & 9.87626 & 40.48731 & $18.8(\mathrm{U})$ & M31N1966-09e ([M75],S10729) & 5.7 & 1 & 2439390 & 1966 \\
\hline 72 & 10.91867 & 41.44963 & 19.3 (B) & M31N1967-09b ([M75],S10732) & 5.3 & 1 & 2439763 & 1967 \\
\hline 73 & 10.71251 & 41.26450 & $18.4(\mathrm{U})$ & M31N1967-11a ([B68],N27) & 0.8 & 1 & 2439802 & 1967 \\
\hline 74 & 11.46352 & 41.54582 & 18.7 (B) & M31N1970-08a ([M75],S10735) & 2.3 & 14 & 2440840 & 1970 \\
\hline 75 & 10.75543 & 41.22672 & $19.3(\mathrm{~B})$ & M31N1971-08a (R091) & 0.9 & 2 & 2441185 & 1971 \\
\hline 76 & 10.73660 & 41.33528 & 17.5 (B) & M31N1972-10a (R095) & 1.1 & -2 & 2441605 & 1972 \\
\hline 77 & 10.72987 & 40.72172 & $18.3(\mathrm{U})$ & M31N1973-09b (ShA1,N14) & 1.7 & 20 & 2441976 & 1973 \\
\hline 78 & 10.74249 & 41.31710 & $18.5(\mathrm{U})$ & M31N1974-07a (R103) & 0.5 & 84 & 2442335 & 1974 \\
\hline 79 & 10.58411 & 41.12401 & $18.4(\mathrm{U})$ & M31N1974-08b (R106) & 0.8 & 57 & 2442335 & 1974 \\
\hline 80 & 10.74133 & 41.42048 & $17.6(\mathrm{U})$ & M31N1978-10a (R120) & 1.1 & 11 & 2443816 & 1978 \\
\hline 81 & 10.87900 & 41.33578 & $18.5(\mathrm{~V})$ & M31N1980-10a (R123) & 0.1 & 3 & 2444523 & 1980 \\
\hline $82(*)$ & 10.57499 & 41.17245 & $18.6(\mathrm{U})$ & M31N1985-09b ([CFN87],N20) & 1.6 & -19 & 2446299 & 1985 \\
\hline 83 & 10.74728 & 41.27864 & 20.4 (B) & M31N1995-11c ([SI2001],N1995-05) & 1.2 & -1 & 2450048 & 1995 \\
\hline 84 & 10.22216 & 40.54756 & 17.8 (B) & M31N1996-12b (ShAl,N54) & 0.9 & -2 & 2450432 & 1996 \\
\hline
\end{tabular}

Note: ${ }^{a}$ Additionally, CFN87 refers to Ciardullo et al. (1987)

there is clearly an object visible on POSS II plates. We checked the possibility of this object being a nova in a globular cluster (similar to the one reported by Quimby et al. 2007), but there is no entry in the catalogue of M 31 globular clusters (Galetti et al. 2006) at this position. Consequently we consider this object not to be a nova in M 31 .

\subsection{Remarks on individual nova candidates}

Here we briefly present remarks on individual objects. We discuss a candidate recurrent nova and a rejected candidate recurrent nova, give details on two novae with large position corrections, and discuss three objects that we detected much earlier than the previously known outbursts. Furthermore, we note a 


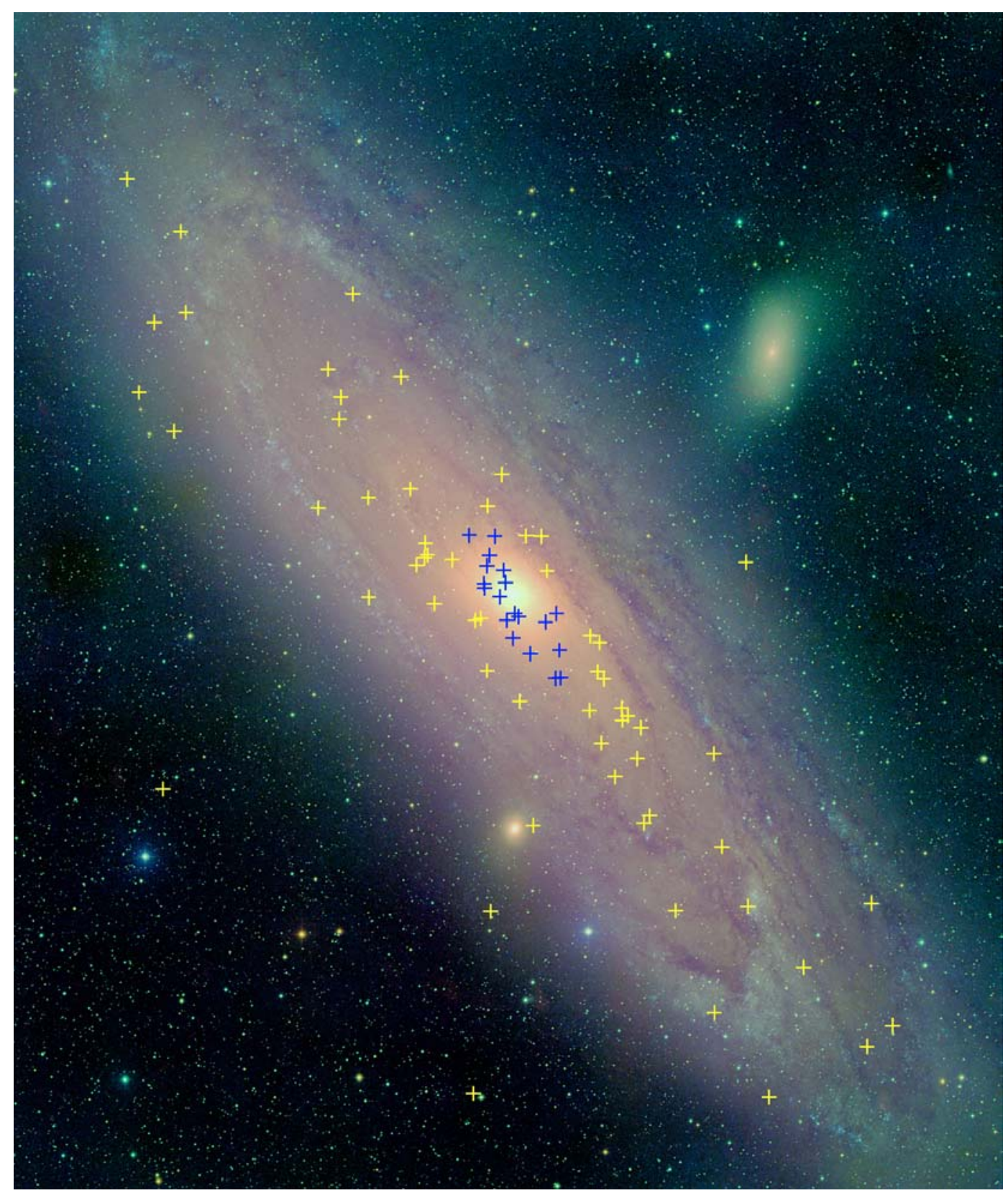

Fig. 7. Distribution of nova candidates found in the present study. Five objects lie outside the displayed field shown by a colour composite based on Tautenburg $U B V$ plates. The field size is $1.7 \times 2: 0$. North is up and east is left. Blue crosses mark nova candidates inside the bulge region with a semi-major axis of $\sim 700^{\prime \prime}$ (e.g. Beaton et al. 2007).

nova that might belong to M32, discuss three novae with lower position accuracy, and give remarks on six very fast or very bright novae and on one very slow nova.

\section{Nova $4=$ M31N 1963-08a $=$ Nova Moffat 2}

Fast nova, $t_{2} \sim 20$ days.

\section{Nova $5=$ M31N 1963-09b = Nova Moffat 1}

Moffat (1967) gave wrong coordinates for this object. In fact, the nova Moffat 1 coincides with nova Rosino 47 (Rosino 1973). This is clearly seen on the plates where the nova candidate originally was found by Moffat. The date of outburst is 2 days earlier than given by Rosino and is very well constrained ( 1 day). This nova is very bright $(15.4 \mathrm{mag})$ and very fast $\left(t_{2} \sim 3\right.$ days $)$.

Nova 7 = M31N 1963-10b = Nova Börngen 8

Fast nova, $t_{2} \sim 13$ days.

Nova $17=$ M31N 1967-10b $=$ Nova Meinunger S10731

Very fast nova, $t_{2} \sim 8$ days.

\section{Nova $18=$ M31N 1967-10c = Nova Börngen 26}

Brightest nova found in this work (15.2 mag). 
Nova 24 = M31N 1973-10b = Nova ShAl 15

Very fast nova, $t_{2} \sim 8$ days.

\section{Nova 28 = M31N 1985-09g = Nova Rosino 139}

We detected this nova 21 days before the outburst date reported in Rosino et al. (1989). But Rosino also noted a possible detection of this nova 2 days before our detection date. Because of the good agreement with our data, let us suppose that this possible detection indeed was real and that Rosino detected the outburst of the nova at nearly the same date as we did.

\section{Nova 31 = M31N 1992-12b = Nova Shafter \& Irby 1992-01}

This nova was found on Tautenburg plates even 84 days before the outburst reported by Shafter \& Irby (2001). First we note that Shafter \& Irby found this nova at the beginning of an observation period with previous observations one year ago. Furthermore, Shafter \& Irby conducted their survey in the $\mathrm{H} \alpha$ band, in which the brightness of novae declines more slowly (Shafter \& Irby 2001). Therefore, we assume that this nova is a slow one and still visible in $\mathrm{H} \alpha 100$ days after outburst. Unfortunately there were no M 31 plates taken in Tautenburg at this time (see Table 18).

\section{Nova $32=$ M31N 1996-08d $=$ Nova ShAl 48}

Despite a position difference of $46^{\prime \prime} 1$, this nova candidate is identified with nova ShAl 48, since the dates of outburst coincide very well and also the finding chart given by Sharov \& Alksnis (1997) evidently shows the same object. In agreement with Alksnis (2006), we assume that the large position difference is due to a typing error in Sharov \& Alksnis (1997).

\section{Nova $40=$ M31N 1972-01b}

Very fast nova, $t_{2} \sim 7$ days.

\section{Nova $43=$ M31N 1975-09a}

This nova candidate was found in our search for recurrent novae using correlations of single-detection objects with the PHS07 catalogue. Originally we identified this object with nova M31N 1999-01a found by Rector et al. (1999), because the distance of the two objects is only 3 ".4. However, a finding chart provided by Rector (2007) clearly shows that both nova candidates have different positions. However, we confirmed this object as a new nova candidate using additional plates.

\section{Nova 44 = M31N 1975-11a}

The position of this newly found candidate correlates (distance 1".0) with nova M31N 1945-09c detected by Baade \& Arp (1964). Hence we classify this nova as recurrent, although the position accuracy of 0 ". 3 given by Baade $\&$ Arp may be too optimistic, and there are no finding charts available for this object to confirm this assumption. Unfortunately Baade \& Arp gave no date of outburst for their nova 32/Table 4. For naming purposes, PHS07 set the date of outburst to 1 September 1945 but the actual date can be between 1945 and 1949. Therefore, the recurrence time is estimated to 26-30 years. As for nova 41, we classify this object as a new nova because the particular outburst in 1975 was not known before.
Nova $57=$ M31N 1962-08a $=$ Nova Meinunger S10730

This candidate is near to the centre of M 32 (distance $\sim 1 ! 8$ ). Therefore, we note that this nova might belong to the galaxy M32.

\section{Nova $58=$ M31N 1962-07a $=$ Nova Börngen 2}

After the detected outburst in September 1962, this object became visible for a year. In agreement with Rosino (1964), we note that this is a very slow nova $\left(t_{2} \sim 350\right.$ days $)$.

\section{Nova $62=$ M31N 1963-10a $=$ Nova Börngen 7}

This object was not detected in the automatic search routine due to the proximity to a bright star. For the same reason, no photometry is given here. We confirmed this nova candidate by eye and computed the coordinates separately. Therefore, the position accuracy is only $\sim 1^{\prime \prime}$.

\section{Nova $64=$ M31N 1964-08b $=$ Nova Börngen 12}

This object was not detected in the automatic search routine because the plates it is detected on are of lower photometric quality and have therefore not been included in the 306 selected plates of the present study. We confirmed this nova candidate by eye and computed the coordinates separately. Therefore, the position accuracy is only $\sim 1$ ". 5 .

\section{Nova $68=$ M31N 1966-09c $=$ Nova Börngen 18}

This object was detected only on broken plates. Therefore, the position accuracy is only $\sim 0$ ". 8

\section{Nova $82=$ M31N 1985-09b = Nova Ciardullo 20}

We detected this nova 19 days before the outburst reported by Ciardullo et al. (1987). Similar to Nova 31, the date of outburst reported by Ciardullo et al. is at the beginning of an observation period of their $\mathrm{H} \alpha$ survey. Therefore, we interpret this object as a moderately fast nova that is visible much longer in the $\mathrm{H} \alpha$ band.

\section{Summary}

Performing a systematic search on a large number of 306 archival Schmidt plates, we found or confirmed in total 84 novae in M31. We found 22 new nova candidates by performing light curve analysis, one of them a candidate for a recurrent nova, 12 nova candidates that are known but not found on Tautenburg plates before, and 21 nova candidates that are known to have been previously discovered on Tautenburg plates. Furthermore, using single detections, we can confirm 29 nova candidates reported in the literature. For all objects we give significantly improved positions. Even though most plates were not taken in the context of a systematic nova survey and the sampling is very irregular, this database has proved to be very valuable by the high number of novae detected on the plates. Moreover, the number of 22 newly-found nova candidates and the fact that one of them is a candidate for a recurrent nova impressively shows the potential of "old" plate archives. Our study was made possible by the ongoing digitization of the Tautenburg plate archive. The re-analysis of archival plates makes it possible to tap the full potential of long-term databases. 
Acknowledgements. We thank Andrejs Alksnis, Halton C. Arp, and Travis Rector for their kind support in finding out details about novae and candidate recurrent novae. We are grateful to Philipp Schalldach for his extensive help in checking and confirming the nova candidates. The anonymous referee is acknowledged for her/his constructive comments and suggestions. This research has made use of the SIMBAD database, the VizieR catalogue access tool, and the Aladin sky atlas, which are operated at the CDS, Strasbourg, France. We acknowledge use of the NASA/IPAC Extragalactic Database (NED), which is operated by the Jet Propulsion Laboratory, California Institute of Technology, under contract with the National Aeronautics and Space Administration. This research has made use of the General catalogue of Variable Stars Volume V Extragalactic Variable Stars (GCVS Vol. V) which is operated at Sternberg Astronomical Institute, Moscow, Russia. We acknowledge use of the Digitized Sky Survey that was produced at the Space Telescope Science Institute under U.S. Government grant NAG W-2166.

\section{References}

Alksnis, A. 2006, private communication

Andruk, V., Kharchenko, N., \& Schilbach, E. 1994, Astron. Nachr. 315, 431

Ansari, R., Auriere, M., Baillon, P., et al. 2004, A\&A, 421, 509

Arp, H. 1956, AJ, 61, 15

Baade, W., \& Arp, H. 1964, ApJ, 139, 1027

Beaton, R. L., Majewski, S. R., Guhathakurta, P., et al. 2007, ApJ, 658, 91

Bertin, E., \& Arnouts S. 1996, A\&AS, 117, 393

Börngen, F. 1968, Mitteilungen des Karl-Schwarzschild-Observatoriums Tautenburg, 40

Brunzendorf, J. 2001, Ph.D. Thesis, Friedrich-Schiller-Universität Jena

Brunzendorf, J., \& Meusinger, H. 1999, A\&AS, 139, 141

Brunzendorf, J., \& Meusinger, H. 2001, A\&A, 373, 38

Ciardullo, R., Ford, H. C., Neill, J. D., et al. 1987, ApJ, 318, 520

Darnley, M. J., Bode, M. F., Kerins, A. M., et al. 2004, MNRAS, 353, 51

Fliri, J., Riffeser, A., Seitz, S., et al. 2006 A\&A, 445, 423
Galleti, S., Federici, L., Bellazzini, M., Buzzoni, A. \& Fusi Pecci, F. 2006, A\&A, 456,985

Greiner, J., Wenzel, W., \& Degel, W. 1990, A\&A, 234, 251

Hatano, K., Branch, D., \& Fisher, A. 1997, ApJ, 487, L45

Henze, M., Meusinger, H., \& Pietsch, W. 2006, IBVS, 5739, 1

Hernanz, M. 2005, in The Astrophysics of Cataclysmic Variables and Related Objects, ed. J.-M. Hameury, \& J.-P. Lasota, ASP Conf. Ser., 330, 265 Hubble, E. 1929, ApJ, 69, 103

Massey, P., Olsen, K. A. G., Hodge, P. W., et al. 2006, AJ, 131, 2478

Meinunger, L. 1975, MitVS, 5, 177

Moffat, A. F. J. 1967, AJ, 72, 10

Monet, D. G., Levine, S. E., Casian, B., et al. 2003, AJ, 125, 984

Ochsenbein, F., Bauer, P., \& Marcout, J. 2000, A\&AS 143, 221

Payne-Gaposchkin, C. 1957, The Galactic Novae, North-Holland, Amsterdam

Pietsch, W., Fliri, J., Freyberg, M. J., et al. 2005, A\&A, 442, 879

Pietsch, W., Haberl, F., Sala, G., et al. 2007, A\&A, 465, 375-392 [PHS07]

Quimby, R., Mondol, P., Wheeler, J. C. , et al. 2007, The Astronomer's Telegram, 1118,1

Rector, T. A. 2007, private communication

Rector, T. A., Jacoby, G. H., Corbett, D. L., \& Denham, M., RBSE Nova Search Team 1999, Bull. Am. Astron. Soc., 31, 1420

Rosino, L. 1964, Ann. d' Astrophys., 27, 498

Rosino, L. 1973, A\&AS, 9, 347

Rosino, L., Cappacioli, M., D‘Onofrio, M., \& Della Valle, M. 1989, AJ, 97, 83

Scholz, R., Meusinger, H., \& Irwin, M. 1997, A\&A, 312, 833

Shafter, A. W., \& Irby, B. K. 2001, ApJ, 563, 749

Sharov, A. S., \& Alksnis, A. 1991, Ap\&SS, 180, 273

Sharov, A. S., \& Alksnis, A. 1992, Ap\&SS, 190, 119

Sharov, A. S., \& Alksnis, A. 1997, AstL, 23, 540

van den Bergh, S. 1964, AJ, 69, 610

Walterbos, R. A. M., \& Kennicutt, R. C. 1987, A\&AS, 69, 311

Warner, B. 1995, Cataclysmic Variable Stars (Cambridge University Press)

Yungelson, L., Livio, M., \& Tutukov, A. 1997, ApJ, 481, 17 


\section{Online Material}


M. Henze et al.: Search for novae in M 31 on digitized Schmidt plates, Online Material p 2

Table 10. Nova light curves continued. See notes below for a more detailed description.

\begin{tabular}{|c|c|c|c|c|c|c|c|c|c|c|c|c|c|}
\hline $\mathrm{PINr}$ & Date & RJD & Limit & Band & $60-11 a$ & $62-08 \mathrm{a}$ & $62-08 b$ & $62-09 \mathrm{a}$ & $62-07 \mathrm{a}$ & $62-09 d$ & $62-09 b$ & $62-11 \mathrm{a}$ & $62-10 a$ \\
\hline 10 & $1960-11-22$ & 37261.34 & 18.5 & $\overline{N F B S}$ & 17.1 & & & & & & & & \\
\hline 35 & $1961-01-12$ & 37312.28 & 19.2 & $N F B S$ & & & & & & & & & \\
\hline 61 & 1961-01-19 & 37319.29 & 18.6 & $N F B S$ & & & & & & & & & \\
\hline 62 & 1961-01-19 & 37319.30 & 18.5 & $N F B S$ & & & & & & & & & \\
\hline 192 & 1961-08-06 & 37517.49 & 19.7 & $N F B S$ & & & & & & & & & \\
\hline 197 & 1961-08-07 & 37519.45 & 19.0 & $N F B S$ & & & & & & & & & \\
\hline 205 & 1961-09-01 & 37544.40 & 19.3 & $N F B S$ & & & & & & & & & \\
\hline 471 & $1962-09-03$ & 37910.55 & 19.5 & $U$ & & 18.9 & 18.8 & 19.5 & 17.8 & & & & \\
\hline 472 & 1962-09-03 & 37910.58 & 19.7 & $B$ & & 19.8 & 19.6 & 20.1 & 19.1 & & & & \\
\hline 490 & 1962-09-05 & 37913.47 & 19.8 & $N F B S$ & & & 19.3 & 19.6 & 18.2 & & & & \\
\hline 669 & $1962-10-23$ & 37961.27 & 19.7 & B & & & & & & 17.5 & & & \\
\hline 717 & 1962-11-19 & 37988.31 & 19.6 & $B$ & & & & & & & 18.4 & 18.8 & \\
\hline 718 & 1962-11-19 & 37988.38 & 17.6 & $U$ & & & & & & & & 17.2 & \\
\hline 721 & $1962-11-20$ & 37989.34 & 19.2 & $U$ & & & & 19.8 & 19.4 & & 17.8 & 18.1 & 18.7 \\
\hline 732 & $1962-11-24$ & 37993.32 & 18.9 & $U$ & & & & & & & 17.9 & 18.4 & \\
\hline 792 & 1963-01-13 & 38043.29 & 17.7 & $N F B S$ & & & & & & & & & \\
\hline 1079 & $1963-08-13$ & 38255.44 & 19.8 & B & & & & & & & & & \\
\hline 1080 & $1963-08-13$ & 38255.46 & 18.7 & V & & & & & & & & & \\
\hline 1085 & 1963-08-14 & 38256.41 & 18.3 & V & & & & & & & & & \\
\hline 1086 & 1963-08-14 & 38256.44 & 19.2 & $B$ & & & & & & & & & \\
\hline 1090 & 1963-08-16 & 38257.50 & 17.9 & $U$ & & & & & & & & & \\
\hline 1092 & 1963-08-17 & 38258.48 & 19.5 & V & & & & & & & & & \\
\hline 1093 & $1963-08-17$ & 38258.55 & 19.0 & $U$ & & & & & & & & & \\
\hline 1096 & $1963-08-20$ & 38261.48 & 19.1 & $B$ & & & & & & & & & \\
\hline 1101 & 1963-08-20 & 38262.46 & 19.5 & $U$ & & & & & 19.6 & & & & \\
\hline 1102 & $1963-08-21$ & 38262.52 & 19.3 & $V$ & & & & & & & & & \\
\hline 1107 & $1963-08-22$ & 38263.52 & 19.5 & $B$ & & & & & & & & & \\
\hline 1108 & $1963-08-22$ & 38263.56 & 19.1 & $U$ & & & & & & & & & \\
\hline 1110 & $1963-08-23$ & 38265.41 & 19.3 & $B$ & & & & & & & & & \\
\hline 1124 & $1963-08-26$ & 38268.45 & 20.1 & $B$ & & & & & & & & & \\
\hline 1125 & $1963-08-27$ & 38268.47 & 19.3 & V & & & & & & & & & \\
\hline 1133 & $1963-08-31$ & 38272.53 & 18.4 & $U$ & & & & & & & & & \\
\hline 1134 & $1963-08-31$ & 38272.58 & 19.0 & $B$ & & & & & & & & & \\
\hline 1135 & $1963-08-31$ & 38272.60 & 17.9 & V & & & & & & & & & \\
\hline 1175 & 1963-09-09 & 38282.37 & 18.6 & V & & & & & & & & & \\
\hline 1181 & 1963-09-10 & 38283.40 & 19.3 & $B$ & & & & & & & & & \\
\hline 1187 & 1963-09-11 & 38284.40 & 19.2 & $U$ & & & & & & & & & \\
\hline 1198 & $1963-09-12$ & 38285.41 & 19.2 & $U$ & & & & & & & & & \\
\hline 1203 & 1963-09-13 & 38286.45 & 19.1 & $U$ & & & & & & & & & \\
\hline 1207 & 1963-09-14 & 38286.62 & 17.4 & V & & & & & & & & & \\
\hline 1212 & 1963-09-15 & 38287.47 & 18.7 & V & & & & & & & & & \\
\hline 1224 & 1963-09-15 & 38288.42 & 19.9 & $B$ & & & & & & & & & \\
\hline 1238 & $1963-09-17$ & 38290.40 & 20.1 & $U$ & & & & & 19.1 & & & & \\
\hline
\end{tabular}

Notes: $\quad{ }^{a}$ : Tautenburg plate number; note that Tables 10-19 list the properties of all 306 plates used in this work.

${ }^{b}$ : Date and Julian Date of plate exposure; RJD = JD - 2400000.

${ }^{c}$ : Limiting magnitude of plate; for a definition of the limiting magnitude in this work see Fig. 2.

${ }^{d}$ : Colour band according to Table 1.

${ }^{e}$ : And subsequent columns give the light curve data; columns are indicated by their M31N 19 name following the naming scheme of PHS07. 
M. Henze et al.: Search for novae in M 31 on digitized Schmidt plates, Online Material p 3

Table 11. Nova light curves continued. See notes below Table 10 for a more detailed description.

\begin{tabular}{|c|c|c|c|c|c|c|c|c|c|c|c|c|c|}
\hline $\mathrm{PINr}$ & Date & RJD & Limit & Band & $63-08 b$ & $63-08 c$ & 63-08a & 63-09a & $63-09 b$ & $63-09 c$ & 63-10b & $63-10 \mathrm{c}$ & $63-10 d$ \\
\hline 1090 & $1963-08-16$ & 38257.50 & 17.9 & $U$ & 17.7 & & & & & & & & \\
\hline 1092 & 1963-08-17 & 38258.48 & 19.5 & $V$ & & & & & & & & & \\
\hline 1093 & 1963-08-17 & 38258.55 & 19.0 & $U$ & 18.3 & 19.4 & & & & & & & \\
\hline 1096 & 1963-08-20 & 38261.48 & 19.1 & $B$ & & & & & & & & & \\
\hline 1101 & 1963-08-20 & 38262.46 & 19.5 & $U$ & 18.5 & 19.1 & 17.2 & & & & & & \\
\hline 1102 & 1963-08-21 & 38262.52 & 19.3 & $V$ & & & & & & & & & \\
\hline 1107 & 1963-08-22 & 38263.52 & 19.5 & $B$ & & & 17.6 & & & & & & \\
\hline 1108 & $1963-08-22$ & 38263.56 & 19.1 & $U$ & 18.8 & 19.7 & 17.1 & & & & & & \\
\hline 1110 & $1963-08-23$ & 38265.41 & 19.3 & $B$ & & & 17.8 & & & & & & \\
\hline 1124 & $1963-08-26$ & 38268.45 & 20.1 & $B$ & & & & & & & & & \\
\hline 1125 & $1963-08-27$ & 38268.47 & 19.3 & $V$ & & & & & & & & & \\
\hline 1133 & 1963-08-31 & 38272.53 & 18.4 & $U$ & 18.6 & & 18.2 & & & & & & \\
\hline 1134 & $1963-08-31$ & 38272.58 & 19.0 & $B$ & & & & & & & & & \\
\hline 1135 & 1963-08-31 & 38272.60 & 17.9 & $V$ & & & & & & & & & \\
\hline 1175 & 1963-09-09 & 38282.37 & 18.6 & $V$ & & & & 18.5 & & & & & \\
\hline 1181 & 1963-09-10 & 38283.40 & 19.3 & $B$ & & & & & & & & & \\
\hline 1187 & 1963-09-11 & 38284.40 & 19.2 & $U$ & & & & 19.1 & 15.4 & & & & \\
\hline 1198 & 1963-09-12 & 38285.41 & 19.2 & $U$ & & & & 19.5 & 16.4 & & & & \\
\hline 1203 & 1963-09-13 & 38286.45 & 19.1 & $U$ & & & & 19.5 & 17.0 & & & & \\
\hline 1207 & 1963-09-14 & 38286.62 & 17.4 & $V$ & & & & & 17.7 & & & & \\
\hline 1212 & 1963-09-15 & 38287.47 & 18.7 & $V$ & & & & & & & & & \\
\hline 1224 & 1963-09-15 & 38288.42 & 19.9 & $B$ & & & & & 18.9 & 18.9 & & & \\
\hline 1238 & 1963-09-17 & 38290.40 & 20.1 & $U$ & & & 19.7 & & 18.5 & 18.0 & & & \\
\hline 1239 & 1963-09-17 & 38290.45 & 18.7 & $V$ & & & & & & & & & \\
\hline 1240 & 1963-09-18 & 38290.46 & 18.5 & $V$ & & & & & & & & & \\
\hline 1254 & 1963-10-08 & 38311.32 & 18.4 & $V$ & & & & & & & 17.2 & & \\
\hline 1260 & $1963-10-12$ & 38315.36 & 18.7 & $U$ & & & & & & & 17.8 & & \\
\hline 1261 & $1963-10-12$ & 38315.43 & 18.3 & $V$ & & & & & & & 17.1 & & \\
\hline 1267 & 1963-10-15 & 38318.40 & 20.4 & $U$ & & & & & & & 17.7 & & \\
\hline 1268 & 1963-10-16 & 38319.37 & 19.9 & $B$ & & & & & & & 18.0 & 20.0 & \\
\hline 1269 & 1963-10-16 & 38319.40 & 19.0 & $V$ & & & & & & & 17.5 & & \\
\hline 1272 & 1963-10-19 & 38322.31 & 19.1 & $U$ & & & & & & & 17.4 & & 19.4 \\
\hline 1275 & 1963-10-20 & 38323.32 & 19.0 & $U$ & & & & & & & 17.6 & & 19.0 \\
\hline 1277 & $1963-10-21$ & 38324.47 & 18.9 & $V$ & & & & & & & 18.3 & & \\
\hline 1278 & $1963-10-22$ & 38324.50 & 18.6 & $V$ & & & & & & & 18.5 & & \\
\hline 1288 & $1963-10-24$ & 38327.43 & 19.5 & $B$ & & & & & & & 18.5 & & \\
\hline 1289 & $1963-10-24$ & 38327.46 & 19.5 & $B$ & & & & & & & 18.5 & & \\
\hline 1290 & 1963-10-25 & 38327.54 & 18.6 & $U$ & & & & & & & 17.7 & & \\
\hline 1295 & 1963-10-25 & 38328.42 & 18.9 & $V$ & & & & & & & 19.0 & & \\
\hline 1296 & $1963-10-25$ & 38328.45 & 19.2 & $V$ & & & & & & & 18.9 & & \\
\hline 1297 & $1963-10-26$ & 38328.52 & 18.8 & $U$ & & & & & & & 17.6 & & \\
\hline 1398 & 1963-12-09 & 38373.22 & 19.0 & $V$ & & & & & & & & & 16.7 \\
\hline 1399 & 1963-12-09 & 38373.26 & 19.0 & $V$ & & & & & & & & & 16.8 \\
\hline 1400 & 1963-12-09 & 38373.33 & 19.0 & $U$ & & & & & & & & & 17.1 \\
\hline 1729 & 1964-09-02 & 38641.43 & 19.9 & $B$ & & & & & & & & & \\
\hline
\end{tabular}


M. Henze et al.: Search for novae in M 31 on digitized Schmidt plates, Online Material p 4

Table 12. Nova light curves continued. See notes below Table 10 for a more detailed description.

\begin{tabular}{|c|c|c|c|c|c|c|c|c|c|c|c|c|c|}
\hline $\mathrm{PINr}$ & Date & RJD & Limit & Band & $63-10 \mathrm{e}$ & $63-12 a$ & $63-12 b$ & 64-09b & 64-09a & 64-10a & $65-06 a$ & $65-09 \mathrm{c}$ & $65-11 \mathrm{c}$ \\
\hline 1278 & $1963-10-22$ & 38324.50 & 18.6 & $V$ & & & & & & & & & \\
\hline 1288 & $1963-10-24$ & 38327.43 & 19.5 & $B$ & 17.7 & & & & & & & & \\
\hline 1289 & $1963-10-24$ & 38327.46 & 19.5 & $B$ & 17.8 & & & & & & & & \\
\hline 1290 & $1963-10-25$ & 38327.54 & 18.6 & $U$ & 17.6 & & & & & & & & \\
\hline 1295 & $1963-10-25$ & 38328.42 & 18.9 & $V$ & 18.4 & & & & & & & & \\
\hline 1296 & $1963-10-25$ & 38328.45 & 19.2 & V & 18.6 & & & & & & & & \\
\hline 1297 & $1963-10-26$ & 38328.52 & 18.8 & $U$ & 17.6 & & & & & & & & \\
\hline 1398 & 1963-12-09 & 38373.22 & 19.0 & V & & 18.0 & 18.6 & & & & & & \\
\hline 1399 & 1963-12-09 & 38373.26 & 19.0 & V & & 18.2 & 18.4 & & & & & & \\
\hline 1400 & $1963-12-09$ & 38373.33 & 19.0 & $U$ & & 18.2 & 18.3 & & & & & & \\
\hline 1729 & 1964-09-02 & 38641.43 & 19.9 & $B$ & & & & 19.5 & & & & & \\
\hline 1735 & 1964-09-03 & 38642.44 & 18.5 & V & & & & 18.9 & & & & & \\
\hline 1736 & 1964-09-04 & 38642.47 & 19.9 & $B$ & & & & 19.2 & & & & & \\
\hline 1750 & 1964-09-13 & 38651.56 & 17.5 & $V$ & & & & 19.9 & 17.3 & & & & \\
\hline 1770 & $1964-09-28$ & 38667.33 & 18.3 & $V$ & & & & 19.6 & & & & & \\
\hline 1771 & 1964-09-28 & 38667.36 & 20.5 & $B$ & & & & & 18.5 & & & & \\
\hline 1772 & $1964-09-28$ & 38667.39 & 18.8 & $V$ & & & & & 18.8 & & & & \\
\hline 1778 & 1964-09-30 & 38669.38 & 19.7 & $B$ & & & & & 18.1 & & & & \\
\hline 1779 & 1964-09-30 & 38669.42 & 19.0 & V & & & & & 18.4 & & & & \\
\hline 1780 & 1964-10-01 & 38669.49 & 19.5 & $B$ & & & & & 18.2 & & & & \\
\hline 1781 & 1964-10-01 & 38669.53 & 18.7 & V & & & & & 18.2 & & & & \\
\hline 1784 & 1964-10-01 & 38670.32 & 18.8 & $U$ & & & & & 17.7 & 18.4 & & & \\
\hline 1786 & 1964-10-01 & 38670.42 & 18.8 & V & & & & & 18.5 & & & & \\
\hline 1788 & 1964-10-02 & 38670.51 & 19.7 & $B$ & & & & & 18.1 & & & & \\
\hline 1789 & 1964-10-02 & 38670.55 & 18.7 & $V$ & & & & & 18.5 & & & & \\
\hline 1790 & 1964-10-02 & 38670.61 & 18.5 & $U$ & & & & & 17.7 & 18.6 & & & \\
\hline 1794 & 1964-10-03 & 38671.51 & 18.1 & V & & & & & & & & & \\
\hline 1795 & 1964-10-03 & 38671.57 & 17.7 & $U$ & & & & & 17.7 & 17.7 & & & \\
\hline 1796 & $1964-10-03$ & 38672.33 & 18.3 & $U$ & & & & & 18.2 & 18.4 & & & \\
\hline 1798 & 1964-10-04 & 38672.55 & 17.5 & $U$ & & & & & 17.9 & & & & \\
\hline 1801 & 1964-10-05 & 38673.56 & 18.4 & $U$ & & & & & 17.9 & 18.8 & & & \\
\hline 1802 & 1964-10-05 & 38674.31 & 18.7 & $U$ & & & & & 17.8 & 19.0 & & & \\
\hline 1803 & 1964-10-05 & 38674.38 & 18.5 & $V$ & & & & & & & & & \\
\hline 1804 & 1964-10-05 & 38674.41 & 20.0 & $B$ & & & & & 18.4 & & & & \\
\hline 1811 & 1964-10-06 & 38674.61 & 18.4 & $U$ & & & & & 17.6 & 18.5 & & & \\
\hline 1812 & $1964-10-06$ & 38675.29 & 19.0 & $U$ & & & & & 17.9 & 18.3 & & & \\
\hline 1813 & $1964-10-06$ & 38675.37 & 18.3 & $U$ & & & & & 17.9 & 18.3 & & & \\
\hline 2047 & 1965-09-04 & 39007.48 & 18.9 & $U$ & & & & & & & 17.6 & & \\
\hline 2068 & $1965-09-21$ & 39025.46 & 18.8 & $U$ & & & & & & & 19.2 & 18.9 & \\
\hline 2140 & $1965-11-23$ & 39088.31 & 18.3 & $V$ & & & & & & & & & 16.5 \\
\hline 2144 & $1965-12-15$ & 39110.32 & 18.5 & $U$ & & & & & & & & & \\
\hline 2256 & 1966-08-19 & 39356.54 & 18.8 & $V$ & & & & & & & & & \\
\hline 2257 & $1966-08-19$ & 39356.56 & 18.5 & $V$ & & & & & & & & & \\
\hline 2315 & $1966-09-20$ & 39389.47 & 19.3 & $U$ & & & & & & & & & \\
\hline
\end{tabular}


M. Henze et al.: Search for novae in M 31 on digitized Schmidt plates, Online Material p 5

Table 13. Nova light curves continued. See notes below Table 10 for a more detailed description.

\begin{tabular}{|c|c|c|c|c|c|c|c|c|c|c|c|c|c|}
\hline PINr & Date & RJD & Limit & Band & $66-09 c$ & $66-09 d$ & $66-09 \mathrm{e}$ & $66-09 f$ & $66-09 b$ & $66-10 b$ & $67-08 a$ & $67-08 b$ & $67-09 b$ \\
\hline 2257 & 1966-08-19 & 39356.56 & 18.5 & $V$ & & & & & & & & & \\
\hline 2315 & 1966-09-20 & 39389.47 & 19.3 & $U$ & 19.5 & 19.8 & 18.8 & 17.5 & 17.2 & & & & \\
\hline 2317 & 1966-09-21 & 39389.59 & 19.3 & $U$ & & 19.4 & 19.1 & 17.7 & 17.5 & & & & \\
\hline 2328 & $1966-09-23$ & 39391.58 & 19.5 & $B$ & & & & & 18.2 & & & & \\
\hline 2329 & $1966-09-23$ & 39391.62 & 19.2 & $B$ & & & & & 18.1 & & & & \\
\hline 2338 & 1966-10-08 & 39407.38 & 19.0 & $B$ & & & & & & & & & \\
\hline 2340 & $1966-10-11$ & 39410.38 & 19.6 & $B$ & & & & & 18.8 & & & & \\
\hline 2341 & 1966-10-11 & 39410.42 & 18.4 & $B$ & & & & & & & & & \\
\hline 2342 & 1966-10-15 & 39414.42 & 18.3 & $B$ & & & & & & & & & \\
\hline 2344 & $1966-10-17$ & 39416.33 & 18.7 & $B$ & & & & & & 18.8 & & & \\
\hline 2345 & 1966-10-17 & 39416.38 & 17.7 & $U$ & & & & & & 18.0 & & & \\
\hline 2346 & 1966-10-18 & 39416.64 & 17.5 & $U$ & & & & & & 17.5 & & & \\
\hline 2348 & 1966-10-20 & 39419.41 & 18.1 & $U$ & & & & & & 17.8 & & & \\
\hline 2526 & $1967-08-28$ & 39731.39 & 18.1 & $B$ & & & & & & & & & \\
\hline 2527 & $1967-08-29$ & 39732.40 & 18.4 & $B$ & & & & & & & & & \\
\hline 2529 & 1967-09-01 & 39734.54 & 19.4 & $B$ & & & & & & & 18.9 & 19.9 & \\
\hline 2530 & 1967-09-01 & 39734.56 & 18.9 & $B$ & & & & & & & 18.7 & & \\
\hline 2531 & 1967-09-01 & 39734.59 & 18.6 & $B$ & & & & & & & & & \\
\hline 2535 & 1967-09-05 & 39739.41 & 19.3 & $B$ & & & & & & & & 19.6 & \\
\hline 2536 & 1967-09-05 & 39739.45 & 19.5 & $B$ & & & & & & & 19.6 & 19.8 & \\
\hline 2537 & 1967-09-06 & 39739.47 & 19.3 & $B$ & & & & & & & 19.6 & 19.5 & \\
\hline 2538 & 1967-09-06 & 39739.50 & 18.9 & $B$ & & & & & & & & & \\
\hline 2558 & 1967-09-29 & 39763.35 & 19.8 & $B$ & & & & & & & & 19.6 & 19.3 \\
\hline 2559 & 1967-09-29 & 39763.38 & 19.1 & $B$ & & & & & & & & & \\
\hline 2560 & 1967-10-01 & 39764.66 & 16.8 & $B$ & & & & & & & & & \\
\hline 2565 & 1967-10-02 & 39765.50 & 19.0 & $B$ & & & & & & & & 20.0 & \\
\hline 2566 & 1967-10-02 & 39765.53 & 19.2 & $B$ & & & & & & & & 20.1 & \\
\hline 2574 & $1967-10-03$ & 39767.37 & 19.0 & $B$ & & & & & & & & & \\
\hline 2576 & 1967-10-04 & 39767.48 & 19.3 & $B$ & & & & & & & & 19.5 & \\
\hline 2588 & 1967-10-11 & 39775.45 & 19.1 & $B$ & & & & & & & & 19.6 & \\
\hline 2590 & $1967-10-12$ & 39775.50 & 19.7 & $B$ & & & & & & & & 19.6 & \\
\hline 2604 & $1967-10-13$ & 39776.52 & 19.0 & $B$ & & & & & & & & & \\
\hline 2605 & 1967-10-13 & 39776.58 & 18.5 & $B$ & & & & & & & & & \\
\hline 2609 & $1967-10-25$ & 39789.28 & 19.1 & $B$ & & & & & & & & 20.1 & \\
\hline 2611 & $1967-10-25$ & 39789.34 & 19.3 & $B$ & & & & & & & & 19.6 & \\
\hline 2615 & $1967-10-26$ & 39790.40 & 19.3 & $B$ & & & & & & & & 19.6 & \\
\hline 2620 & $1967-10-29$ & 39792.51 & 19.4 & $B$ & & & & & & & & 19.8 & \\
\hline 2623 & $1967-10-29$ & 39793.42 & 19.2 & $B$ & & & & & & & & 19.5 & \\
\hline 2624 & $1967-10-29$ & 39793.45 & 18.9 & $B$ & & & & & & & & 19.5 & \\
\hline 2631 & 1967-10-30 & 39794.47 & 19.2 & $B$ & & & & & & & & 19.7 & \\
\hline 2632 & 1967-10-31 & 39794.49 & 18.9 & $B$ & & & & & & & & & \\
\hline 2636 & 1967-11-01 & 39796.31 & 17.5 & $B$ & & & & & & & & & \\
\hline 2638 & 1967-11-03 & 39797.55 & 19.4 & $B$ & & & & & & & & 19.5 & \\
\hline 2639 & $1967-11-03$ & 39797.57 & 18.6 & $B$ & & & & & & & & & \\
\hline 2644 & $1967-11-03$ & 39798.42 & 19.5 & $B$ & & & & & & & & 19.6 & \\
\hline 2645 & 1967-11-03 & 39798.45 & 19.4 & $B$ & & & & & & & & 19.7 & \\
\hline 2652 & 1967-11-06 & 39800.57 & 19.8 & $B$ & & & & & & & & 19.8 & \\
\hline 2653 & 1967-11-06 & 39800.59 & 18.1 & $B$ & & & & & & & & & \\
\hline 2661 & 1967-11-06 & 39801.45 & 19.5 & $B$ & & & & & & & & 19.9 & \\
\hline 2662 & 1967-11-07 & 39801.48 & 19.6 & $B$ & & & & & & & & 19.7 & \\
\hline 2670 & 1967-11-07 & 39802.37 & 19.5 & $B$ & & & & & & & & 19.5 & \\
\hline 2671 & 1967-11-07 & 39802.39 & 19.4 & $B$ & & & & & & & & 19.5 & \\
\hline 2672 & 1967-11-07 & 39802.43 & 19.2 & $U$ & & & & & & & & & \\
\hline
\end{tabular}


M. Henze et al.: Search for novae in M 31 on digitized Schmidt plates, Online Material p 6

Table 14. Nova light curves continued. See notes below Table 10 for a more detailed description.

\begin{tabular}{|c|c|c|c|c|c|c|c|c|c|c|c|c|c|}
\hline $\mathrm{PlNr}$ & Date & RJD & Limit & Band & $67-09 a$ & $67-10 a$ & $67-10 b$ & $67-10 \mathrm{c}$ & $67-10 \mathrm{~d}$ & $67-11 a$ & $69-10 a$ & $70-08 a$ & $70-11 a$ \\
\hline 2538 & 1967-09-06 & 39739.50 & 18.9 & $B$ & & & & & & & & & \\
\hline 2558 & $1967-09-29$ & 39763.35 & 19.8 & $B$ & 19.7 & & & & & & & & \\
\hline 2559 & $1967-09-29$ & 39763.38 & 19.1 & $B$ & & & & & & & & & \\
\hline 2560 & $1967-10-01$ & 39764.66 & 16.8 & $B$ & & & & & & & & & \\
\hline 2565 & $1967-10-02$ & 39765.50 & 19.0 & $B$ & 19.2 & & & & & & & & \\
\hline 2566 & $1967-10-02$ & 39765.53 & 19.2 & $B$ & & & & & & & & & \\
\hline 2574 & $1967-10-03$ & 39767.37 & 19.0 & $B$ & 19.0 & & & & & & & & \\
\hline 2576 & 1967-10-04 & 39767.48 & 19.3 & $B$ & 19.3 & & & & & & & & \\
\hline 2588 & 1967-10-11 & 39775.45 & 19.1 & $B$ & & 19.0 & & & & & & & \\
\hline 2590 & $1967-10-12$ & 39775.50 & 19.7 & $B$ & & 18.0 & & & & & & & \\
\hline 2604 & $1967-10-13$ & 39776.52 & 19.0 & $B$ & & 18.0 & & & & & & & \\
\hline 2605 & $1967-10-13$ & 39776.58 & 18.5 & $B$ & & 17.5 & & & & & & & \\
\hline 2609 & $1967-10-25$ & 39789.28 & 19.1 & $B$ & & 17.7 & 17.0 & & & & & & \\
\hline 2611 & $1967-10-25$ & 39789.34 & 19.3 & $B$ & & 17.9 & 16.9 & & & & & & \\
\hline 2615 & 1967-10-26 & 39790.40 & 19.3 & $B$ & & 17.9 & 17.5 & & & & & & \\
\hline 2620 & $1967-10-29$ & 39792.51 & 19.4 & $B$ & & 18.4 & 18.0 & & & & & & \\
\hline 2623 & $1967-10-29$ & 39793.42 & 19.2 & $B$ & & 17.3 & 18.1 & 15.2 & & & & & \\
\hline 2624 & 1967-10-29 & 39793.45 & 18.9 & $B$ & & 17.3 & 18.2 & 15.2 & & & & & \\
\hline 2631 & 1967-10-30 & 39794.47 & 19.2 & $B$ & & 18.1 & 18.6 & 15.2 & & & & & \\
\hline 2632 & $1967-10-31$ & 39794.49 & 18.9 & $B$ & & 18.0 & 18.5 & 15.4 & & & & & \\
\hline 2636 & $1967-11-01$ & 39796.31 & 17.5 & $B$ & & & & 15.4 & & & & & \\
\hline 2638 & 1967-11-03 & 39797.55 & 19.4 & $B$ & & 18.1 & 19.2 & 15.6 & 18.7 & & & & \\
\hline 2639 & 1967-11-03 & 39797.57 & 18.6 & $B$ & & 18.5 & & 15.6 & & & & & \\
\hline 2644 & 1967-11-03 & 39798.42 & 19.5 & $B$ & 19.8 & 18.5 & 19.2 & 15.7 & 18.6 & & & & \\
\hline 2645 & 1967-11-03 & 39798.45 & 19.4 & $B$ & 19.8 & 18.7 & 18.8 & 15.7 & & & & & \\
\hline 2652 & 1967-11-06 & 39800.57 & 19.8 & $B$ & 19.4 & & 19.3 & 15.8 & & 18.1 & & & \\
\hline 2653 & $1967-11-06$ & 39800.59 & 18.1 & $B$ & & 18.2 & & 15.7 & & & & & \\
\hline 2661 & 1967-11-06 & 39801.45 & 19.5 & $B$ & & 18.5 & & 15.6 & & & & & \\
\hline 2662 & 1967-11-07 & 39801.48 & 19.6 & $B$ & 19.7 & 18.1 & 19.4 & 15.7 & & & & & \\
\hline 2670 & 1967-11-07 & 39802.37 & 19.5 & $B$ & & 18.2 & 19.9 & 15.7 & 19.2 & & & & \\
\hline 2671 & 1967-11-07 & 39802.39 & 19.4 & $B$ & & 18.7 & 19.6 & 15.6 & 19.1 & & & & \\
\hline 2672 & 1967-11-07 & 39802.43 & 19.2 & $U$ & 19.3 & 17.5 & 19.0 & 15.1 & 18.4 & 18.4 & & & \\
\hline 2677 & 1967-11-09 & 39804.45 & 17.6 & $B$ & & & & 15.8 & & & & & \\
\hline 2678 & $1967-11-10$ & 39804.49 & 18.0 & $B$ & & & & 15.9 & & & & & \\
\hline 2915 & $1969-07-25$ & 40427.53 & 18.3 & $B$ & & & & & & & & & \\
\hline 2923 & $1969-10-04$ & 40499.35 & 19.6 & V & & & & & & & & & \\
\hline 2928 & 1969-10-04 & 40499.42 & 19.6 & $V$ & & & & & & & & & \\
\hline 2934 & 1969-10-05 & 40500.36 & 18.8 & $V$ & & & & & & & & & \\
\hline 2936 & 1969-10-05 & 40500.42 & 19.0 & $V$ & & & & & & & & & \\
\hline 2946 & 1969-10-07 & 40501.50 & 19.0 & $V$ & & & & & & & & & \\
\hline 2947 & $1969-10-07$ & 40501.52 & 19.2 & $V$ & & & & & & & & & \\
\hline 2957 & $1969-10-08$ & 40503.39 & 18.4 & $B$ & & & & & & & & & \\
\hline 2958 & $1969-10-08$ & 40503.41 & 19.3 & $B$ & & & & & & & & & \\
\hline 2968 & 1969-10-09 & 40504.37 & 19.5 & $B$ & & & & & & & & & \\
\hline 2969 & 1969-10-09 & 40504.39 & 19.8 & $B$ & & & & & & & & & \\
\hline 2985 & $1969-10-11$ & 40506.40 & 19.0 & V & & & & & & & & & \\
\hline 2990 & $1969-10-13$ & 40508.35 & 19.1 & $N F B S$ & & & & & & & & & \\
\hline 2994 & $1969-10-13$ & 40508.42 & 19.5 & B & & & & & & & 18.4 & & \\
\hline 2995 & $1969-10-13$ & 40508.44 & 18.7 & $B$ & & & & & & & & & \\
\hline 2997 & $1969-10-14$ & 40508.48 & 19.4 & $N F B S$ & & & & & & & 18.2 & & \\
\hline 3013 & 1970-02-06 & 40624.26 & 18.7 & B & & & & & & & & & \\
\hline 3014 & 1970-02-06 & 40624.29 & 18.8 & $B$ & & & & & & & & & \\
\hline 3024 & 1970-02-09 & 40627.27 & 18.6 & $B$ & & & & & & & & & \\
\hline 3035 & 1970-03-03 & 40649.30 & 18.9 & $B$ & & & & & & & & & \\
\hline 3059 & 1970-03-09 & 40655.29 & 17.8 & $B$ & & & & & & & & & \\
\hline 3138 & $1970-09-10$ & 40839.58 & 19.0 & $B$ & & & & & & & & 18.7 & \\
\hline 3139 & 1970-09-10 & 40839.61 & 18.3 & $B$ & & & & & & & & & \\
\hline 3171 & $1970-11-25$ & 40916.22 & 18.9 & $B$ & & & & & & & & & 18.9 \\
\hline 3172 & $1970-11-25$ & 40916.25 & 19.5 & $B$ & & & & & & & & & 19.1 \\
\hline 3190 & $1970-11-26$ & 40917.29 & 19.0 & $B$ & & & & & & & & & 18.8 \\
\hline 3191 & 1970-11-26 & 40917.32 & 19.2 & $B$ & & & & & & & & & 19.1 \\
\hline 3224 & $1971-08-21$ & 41184.60 & 18.8 & $B$ & & & & & & & & & \\
\hline
\end{tabular}


M. Henze et al.: Search for novae in M31 on digitized Schmidt plates, Online Material $p 7$

Table 15. Nova light curves continued. See notes below Table 10 for a more detailed description.

\begin{tabular}{|c|c|c|c|c|c|c|c|c|c|c|c|c|c|}
\hline PINr & Date & RJD & Limit & Band & $71-08 a$ & $72-01 b$ & 72-10a & $72-11 a$ & $72-12 b$ & 73-10a & 73-10b & $73-09 b$ & $74-08 \mathrm{a}$ \\
\hline 3191 & $1970-11-26$ & 40917.32 & 19.2 & $B$ & & & & & & & & & \\
\hline 3224 & 1971-08-21 & 41184.60 & 18.8 & $B$ & 19.3 & & & & & & & & \\
\hline 3243 & $1971-08-29$ & 41193.45 & 19.9 & $B$ & & & & & & & & & \\
\hline 3244 & 1971-08-30 & 41193.51 & 18.6 & $U$ & & & & & & & & & \\
\hline 3245 & 1971-08-30 & 41193.56 & 20.0 & $B$ & & & & & & & & & \\
\hline 3253 & $1971-09-22$ & 41217.41 & 19.0 & $U$ & & & & & & & & & \\
\hline 3254 & 1971-09-23 & 41217.51 & 19.7 & $B$ & & & & & & & & & \\
\hline 3255 & $1971-09-23$ & 41217.56 & 19.1 & $U$ & & & & & & & & & \\
\hline 3256 & $1971-09-23$ & 41217.63 & 19.7 & $B$ & & & & & & & & & \\
\hline 3263 & 1971-11-17 & 41273.43 & 18.0 & $B$ & & & & & & & & & \\
\hline 3267 & 1971-11-20 & 41275.56 & 18.0 & $B$ & & & & & & & & & \\
\hline 3272 & 1971-11-20 & 41276.25 & 19.4 & $B$ & & & & & & & & & \\
\hline 3273 & 1971-11-20 & 41276.27 & 19.1 & $B$ & & & & & & & & & \\
\hline 3274 & 1971-11-20 & 41276.30 & 17.7 & $U$ & & & & & & & & & \\
\hline 3275 & 1971-11-20 & 41276.38 & 18.6 & $B$ & & & & & & & & & \\
\hline 3277 & $1971-11-23$ & 41278.51 & 19.0 & $B$ & & & & & & & & & \\
\hline 3282 & 1972-01-11 & 41328.40 & 17.1 & $B$ & & 16.7 & & & & & & & \\
\hline 3300 & 1972-01-15 & 41332.24 & 18.2 & $U$ & & 16.9 & & & & & & & \\
\hline 3309 & 1972-01-16 & 41333.22 & 18.4 & $B$ & & 17.8 & & & & & & & \\
\hline 3315 & 1972-01-17 & 41334.23 & 17.5 & $B$ & & & & & & & & & \\
\hline 3319 & 1972-01-18 & 41335.23 & 18.7 & $B$ & & 18.7 & & & & & & & \\
\hline 3333 & $1972-01-19$ & 41336.26 & 18.3 & $B$ & & & & & & & & & \\
\hline 3339 & 1972-01-20 & 41337.32 & 17.8 & $U$ & & & & & & & & & \\
\hline 3673 & 1972-10-14 & 41605.43 & 19.2 & $B$ & & & 17.5 & 18.6 & & & & & \\
\hline 3675 & $1972-10-30$ & 41621.45 & 19.3 & $B$ & & & & 18.5 & & & & & \\
\hline 3676 & 1972-10-31 & 41621.48 & 18.9 & $B$ & & & & 18.6 & & & & & \\
\hline 3680 & 1972-11-01 & 41623.31 & 19.3 & $B$ & & & & 18.9 & & & & & \\
\hline 3703 & 1972-11-14 & 41635.52 & 19.0 & $B$ & & & & & & & & & \\
\hline 3734 & $1972-12-14$ & 41665.50 & 16.6 & $B$ & & & & & & & & & \\
\hline 3746 & $1972-12-28$ & 41680.28 & 19.2 & $B$ & & & & & 18.8 & & & & \\
\hline 3758 & $1972-12-29$ & 41681.34 & 18.5 & $B$ & & & & & 19.0 & & & & \\
\hline 3826 & 1973-10-20 & 41976.33 & 18.6 & $U$ & & & & & & 18.5 & 18.2 & 18.3 & \\
\hline 3827 & 1973-10-20 & 41976.38 & 19.3 & $B$ & & & & & & 20.0 & 17.7 & 20.2 & \\
\hline 3830 & 1973-10-24 & 41980.33 & 19.2 & $B$ & & & & & & & 16.1 & & \\
\hline 3837 & $1973-10-27$ & 41982.48 & 19.7 & $B$ & & & & & & & 16.8 & & \\
\hline 3838 & $1973-10-27$ & 41982.52 & 19.8 & $B$ & & & & & & 19.5 & 17.4 & & \\
\hline 3839 & $1973-10-27$ & 41982.56 & 19.4 & $B$ & & & & & & & 17.0 & & \\
\hline 3840 & $1973-10-27$ & 41982.59 & 18.4 & $B$ & & & & & & & 16.9 & & \\
\hline 3846 & 1973-10-27 & 41983.42 & 18.5 & V & & & & & & & 16.5 & & \\
\hline 3849 & $1973-10-28$ & 41983.50 & 19.5 & $B$ & & & & & & 18.9 & 17.3 & & \\
\hline 3861 & $1973-10-28$ & 41984.42 & 18.5 & $V$ & & & & & & & 16.9 & & \\
\hline 3868 & 1973-10-31 & 41987.43 & 19.3 & $B$ & & & & & & 19.7 & 18.3 & 19.4 & \\
\hline 3870 & 1973-11-01 & 41987.53 & 18.8 & $B$ & & & & & & & 18.1 & & \\
\hline 3872 & 1973-11-01 & 41987.60 & 18.8 & $B$ & & & & & & & 18.0 & & \\
\hline 3907 & $1973-11-21$ & 42008.43 & 18.7 & $B$ & & & & & & & & & \\
\hline 3953 & $1973-12-19$ & 42036.33 & 18.4 & $B$ & & & & & & & & & \\
\hline 3958 & $1973-12-20$ & 42037.33 & 19.0 & $B$ & & & & & & & & 18.9 & \\
\hline 4172 & $1974-08-16$ & 42275.51 & 18.6 & $B$ & & & & & & & & & 18.3 \\
\hline 4175 & 1974-08-16 & 42275.58 & 18.9 & $B$ & & & & & & & & & 18.7 \\
\hline 4188 & $1974-08-23$ & 42282.54 & 18.8 & $B$ & & & & & & & & & \\
\hline
\end{tabular}


M. Henze et al.: Search for novae in M 31 on digitized Schmidt plates, Online Material p 8

Table 16. Nova light curves continued. See notes below Table 10 for a more detailed description.

\begin{tabular}{|c|c|c|c|c|c|c|c|c|c|c|c|c|c|}
\hline PINr & Date & RJD & Limit & Band & $74-08 b$ & $74-07 a$ & $75-02 a$ & $75-09 a$ & $75-10 a$ & $75-11 a$ & $75-12 b$ & $78-10 a$ & $79-11 a$ \\
\hline 4175 & $1974-08-16$ & 42275.58 & 18.9 & $B$ & & & & & & & & & \\
\hline 4188 & $1974-08-23$ & 42282.54 & 18.8 & $B$ & 18.3 & & & & & & & & \\
\hline 4196 & 1974-09-11 & 42302.42 & 19.8 & $B$ & & & & & & & & & \\
\hline 4197 & 1974-09-11 & 42302.45 & 20.6 & $B$ & & & & & & & & & \\
\hline 4198 & $1974-09-12$ & 42302.48 & 19.2 & V & & & & & & & & & \\
\hline 4209 & $1974-10-14$ & 42334.51 & 18.3 & $U$ & 18.4 & 18.5 & & & & & & & \\
\hline 4289 & $1975-02-10$ & 42454.25 & 19.0 & $B$ & & & 15.7 & & & & & & \\
\hline 4294 & $1975-02-11$ & 42455.28 & 18.4 & $B$ & & & 15.6 & & & & & & \\
\hline 4429 & 1975-08-04 & 42628.56 & 19.3 & $B$ & & & & & & & & & \\
\hline 4506 & 1975-09-09 & 42665.40 & 19.3 & $B$ & & & & 18.5 & & & & & \\
\hline 4511 & $1975-09-29$ & 42685.42 & 19.6 & $B$ & & & & & & & & & \\
\hline 4512 & $1975-09-29$ & 42685.45 & 19.7 & $B$ & & & & & & & & & \\
\hline 4518 & $1975-10-11$ & 42696.48 & 18.3 & V & & & & & & & & & \\
\hline 4528 & $1975-10-29$ & 42715.41 & 19.1 & $B$ & & & & & & & & & \\
\hline 4532 & $1975-10-30$ & 42716.31 & 18.5 & $V$ & & & & & & & & & \\
\hline 4533 & $1975-10-30$ & 42716.33 & 19.6 & $B$ & & & & & 19.4 & & & & \\
\hline 4534 & $1975-10-30$ & 42716.35 & 18.9 & $B$ & & & & & & & & & \\
\hline 4537 & $1975-10-30$ & 42716.46 & 18.9 & $V$ & & & & & & & & & \\
\hline 4545 & $1975-10-31$ & 42717.26 & 19.1 & $B$ & & & & & & & & & \\
\hline 4547 & $1975-10-31$ & 42717.30 & 18.7 & V & & & & & & & & & \\
\hline 4550 & $1975-10-31$ & 42717.36 & 18.8 & V & & & & & & & & & \\
\hline 4560 & $1975-11-01$ & 42718.26 & 19.4 & $B$ & & & & & 19.1 & & & & \\
\hline 4563 & 1975-11-02 & 42719.27 & 19.3 & $B$ & & & & & & & & & \\
\hline 4572 & $1975-11-24$ & 42741.25 & 19.2 & V & & & & & & 18.6 & & & \\
\hline 4575 & $1975-11-24$ & 42741.33 & 18.6 & $B$ & & & & & & 18.7 & & & \\
\hline 4578 & $1975-11-24$ & 42741.39 & 18.0 & V & & & & & & & & & \\
\hline 4579 & $1975-11-25$ & 42742.22 & 18.9 & $B$ & & & & & & & & & \\
\hline 4580 & $1975-11-25$ & 42742.24 & 18.3 & V & & & & & & & & & \\
\hline 4581 & $1975-11-25$ & 42742.26 & 18.2 & V & & & & & & & & & \\
\hline 4584 & $1975-11-25$ & 42742.32 & 18.7 & V & & & & & & 18.9 & & & \\
\hline 4610 & $1975-12-28$ & 42775.22 & 18.5 & $V$ & & & & & & & & & \\
\hline 4611 & $1975-12-28$ & 42775.24 & 18.8 & V & & & & & & & & & \\
\hline 4612 & $1975-12-28$ & 42775.26 & 19.4 & $B$ & & & & & & & 18.6 & & \\
\hline 4613 & $1975-12-28$ & 42775.28 & 19.4 & $B$ & & & & & & & 18.7 & & \\
\hline 4614 & $1975-12-28$ & 42775.31 & 18.4 & $U$ & & & & & & & 17.7 & & \\
\hline 4615 & $1975-12-28$ & 42775.35 & 18.7 & $U$ & & & & & & & 17.7 & & \\
\hline 4651 & $1976-02-02$ & 42811.28 & 18.2 & $B$ & & & & & & & & & \\
\hline 4824 & $1976-07-30$ & 42989.53 & 19.4 & $B$ & & & & & & & & & \\
\hline 4889 & $1976-09-23$ & 43044.53 & 19.4 & $B$ & & & & & & & & & \\
\hline 4898 & $1976-10-21$ & 43073.43 & 19.3 & $B$ & & & & & & & & & \\
\hline 5200 & 1978-11-03 & 43816.27 & 18.1 & $U$ & & & & & & & & 17.6 & \\
\hline 5391 & $1979-10-26$ & 44172.50 & 18.5 & $B$ & & & & & & & & & \\
\hline 5392 & $1979-10-26$ & 44172.53 & 17.2 & $U$ & & & & & & & & & \\
\hline 5410 & 1979-11-16 & 44194.39 & 19.0 & $B$ & & & & & & & & & 19.0 \\
\hline 5411 & $1979-11-16$ & 44194.41 & 18.5 & $U$ & & & & & & & & & 18.4 \\
\hline 5414 & $1979-12-14$ & 44222.35 & 19.5 & $B$ & & & & & & & & & 19.6 \\
\hline 5428 & $1980-01-14$ & 44253.30 & 19.0 & $B$ & & & & & & & & & 19.2 \\
\hline 5547 & 1980-09-07 & 44489.52 & 19.1 & $B$ & & & & & & & & & \\
\hline
\end{tabular}


M. Henze et al.: Search for novae in M31 on digitized Schmidt plates, Online Material $p 9$

Table 17. Nova light curves continued. See notes below Table 10 for a more detailed description.

\begin{tabular}{|c|c|c|c|c|c|c|c|c|c|c|c|c|c|}
\hline $\mathrm{PINr}$ & Date & RJD & Limit & Band & $80-09 b$ & $80-09 a$ & $80-09 \mathrm{c}$ & $80-10 a$ & $84-08 a$ & $85-08 b$ & $85-08 \mathrm{a}$ & $85-09 \mathrm{~g}$ & $85-09 b$ \\
\hline 5428 & 1980-01-14 & 44253.30 & 19.0 & $B$ & & & & & & & & & \\
\hline 5547 & 1980-09-07 & 44489.52 & 19.1 & $B$ & 19.0 & 17.7 & & & & & & & \\
\hline 5548 & 1980-09-07 & 44489.54 & 18.2 & $U$ & 18.0 & 17.6 & 18.0 & & & & & & \\
\hline 5560 & 1980-09-07 & 44490.45 & 18.4 & $U$ & 18.2 & 18.0 & 18.1 & & & & & & \\
\hline 5595 & $1980-10-10$ & 44523.33 & 19.2 & V & & & & 18.5 & & & & & \\
\hline 5606 & 1980-10-31 & 44544.34 & 18.7 & $B$ & & & & & & & & & \\
\hline 5608 & 1980-11-01 & 44545.33 & 19.1 & $B$ & & & & & & & & & \\
\hline 5843 & $1982-08-15$ & 45196.49 & 18.5 & $B$ & & & & & & & & & \\
\hline 6040 & 1984-07-30 & 45911.52 & 18.8 & $N F B S$ & & & & & & & & & \\
\hline 6045 & 1984-08-01 & 45913.50 & 19.2 & $N F B S$ & & & & & & & & & \\
\hline 6055 & 1984-08-27 & 45940.45 & 19.6 & B & & & & & 19.3 & & & & \\
\hline 6057 & $1984-08-28$ & 45940.52 & 19.6 & $B$ & & & & & 19.5 & & & & \\
\hline 6061 & 1984-09-28 & 45971.51 & 19.0 & $N F B S$ & & & & & & & & & \\
\hline 6063 & 1984-09-28 & 45971.55 & 19.0 & $N F B S$ & & & & & & & & & \\
\hline 6125 & 1985-08-21 & 46299.45 & 19.5 & B & & & & & & 19.1 & 19.3 & 19.4 & \\
\hline 6126 & $1985-08-22$ & 46299.48 & 19.5 & $U$ & & & & & & 18.5 & 18.6 & 18.4 & 18.6 \\
\hline 6132 & $1985-08-24$ & 46301.50 & 19.6 & $B$ & & & & & & 19.5 & 19.7 & 19.2 & \\
\hline 6133 & $1985-08-24$ & 46301.54 & 19.0 & $U$ & & & & & & 18.6 & 19.1 & 18.2 & 19.2 \\
\hline 6137 & $1985-09-13$ & 46321.54 & 19.8 & $B$ & & & & & & 20.4 & 19.9 & 19.9 & \\
\hline 6138 & $1985-09-13$ & 46321.58 & 18.9 & $U$ & & & & & & & 19.1 & 19.0 & \\
\hline 6277 & 1986-09-10 & 46683.47 & 19.5 & $N F B S$ & & & & & & & & & \\
\hline
\end{tabular}

Table 18. Nova light curves continued. See notes below Table 10 for a more detailed description.

\begin{tabular}{|c|c|c|c|c|c|c|c|c|c|c|c|c|c|}
\hline PINr & Date & RJD & Limit & Band & $85-08 \mathrm{c}$ & $85-09 h$ & $87-08 \mathrm{a}$ & $92-12 b$ & $92-09 a$ & $95-11 \mathrm{c}$ & $96-08 \mathrm{~g}$ & $96-08 \mathrm{e}$ & $96-08 \mathrm{~d}$ \\
\hline 6125 & $1985-08-21$ & 46299.45 & 19.5 & $B$ & & & & & & & & & \\
\hline 6126 & $1985-08-22$ & 46299.48 & 19.5 & $U$ & 18.8 & & & & & & & & \\
\hline 6132 & $1985-08-24$ & 46301.50 & 19.6 & $B$ & 20.5 & & & & & & & & \\
\hline 6133 & $1985-08-24$ & 46301.54 & 19.0 & $U$ & 19.0 & & & & & & & & \\
\hline 6137 & 1985-09-13 & 46321.54 & 19.8 & $B$ & & 17.9 & & & & & & & \\
\hline 6138 & 1985-09-13 & 46321.58 & 18.9 & $U$ & & 17.4 & & & & & & & \\
\hline 6277 & 1986-09-10 & 46683.47 & 19.5 & $N F B S$ & & & & & & & & & \\
\hline 6278 & 1986-09-10 & 46683.49 & 19.7 & $N F B S$ & & & & & & & & & \\
\hline 6379 & 1986-11-29 & 46764.45 & 18.9 & B & & & & & & & & & \\
\hline 6518 & 1987-08-31 & 47039.43 & 19.9 & $B$ & & & 17.9 & & & & & & \\
\hline 6523 & 1987-09-04 & 47042.57 & 19.9 & $B$ & & & 19.0 & & & & & & \\
\hline 8089 & 1992-08-06 & 48840.51 & 20.0 & $B$ & & & & & & & & & \\
\hline 8095 & 1992-08-27 & 48862.44 & 19.3 & $B$ & & & & & & & & & \\
\hline 8149 & $1992-09-25$ & 48891.39 & 19.3 & $B$ & & & & 18.3 & & & & & \\
\hline 8171 & 1992-09-28 & 48893.49 & 20.2 & $B$ & & & & 18.0 & 16.5 & & & & \\
\hline 8183 & $1992-09-29$ & 48894.47 & 19.9 & $B$ & & & & 18.3 & 17.4 & & & & \\
\hline 8840 & $1995-01-27$ & 49745.30 & 17.9 & $B$ & & & & & & & & & \\
\hline 9090 & $1995-11-26$ & 50048.34 & 19.8 & $B$ & & & & & & 20.4 & & & \\
\hline 9145 & 1996-08-20 & 50315.51 & 19.4 & $B$ & & & & & & & 17.3 & 19.5 & 17.3 \\
\hline 9150 & $1996-08-21$ & 50316.53 & 19.5 & $B$ & & & & & & & 16.4 & 19.5 & 17.8 \\
\hline 9162 & $1996-12-14$ & 50432.35 & 18.7 & $B$ & & & & & & & & & \\
\hline
\end{tabular}

Table 19. Nova light curves continued. See notes below Table 10 for a more detailed description.

\begin{tabular}{rrrrcc}
\hline \hline $\mathrm{PINr}$ & Date & RJD & Limit & Band & $96-12 \mathrm{~b}$ \\
\hline 9150 & $1996-08-21$ & 50316.53 & 19.5 & $B$ & \\
9162 & $1996-12-14$ & 50432.35 & 18.7 & $B$ & 17.8 \\
\hline
\end{tabular}


M. Henze et al.: Search for novae in M31 on digitized Schmidt plates, Online Material p 10

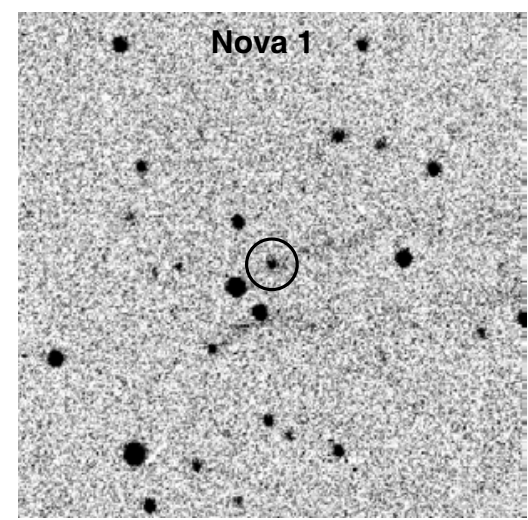

(a) Nova 1 (B)

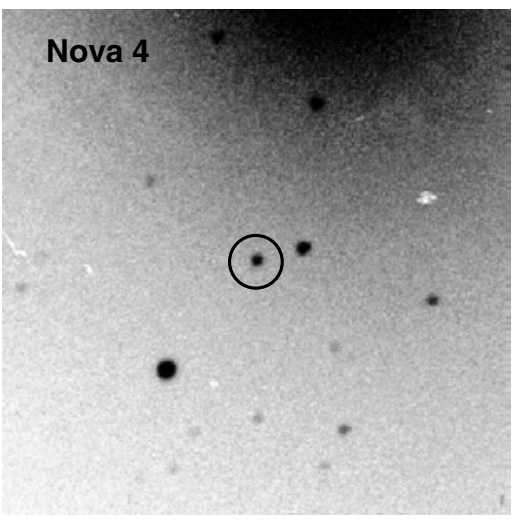

(d) Nova 4 (U)

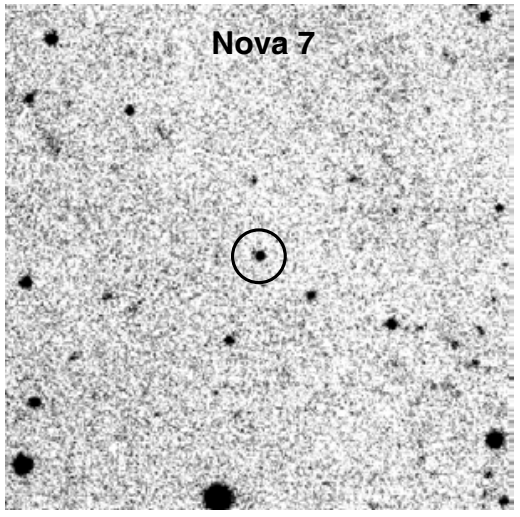

(g) Nova 7 (B)

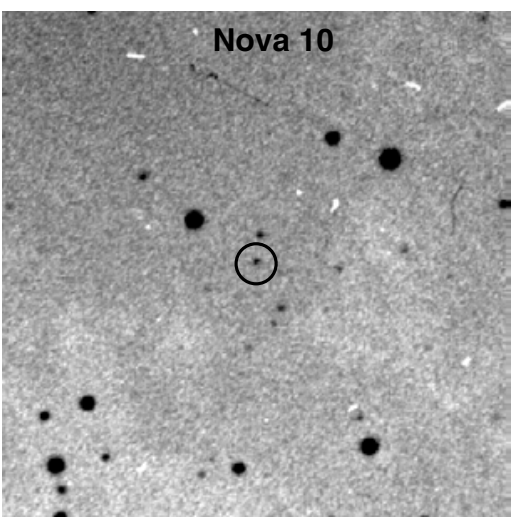

(j) Nova $10(\mathrm{U})$

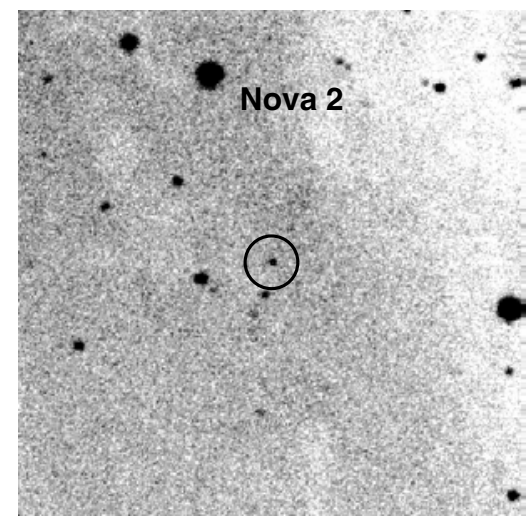

(b) Nova 2 (B)

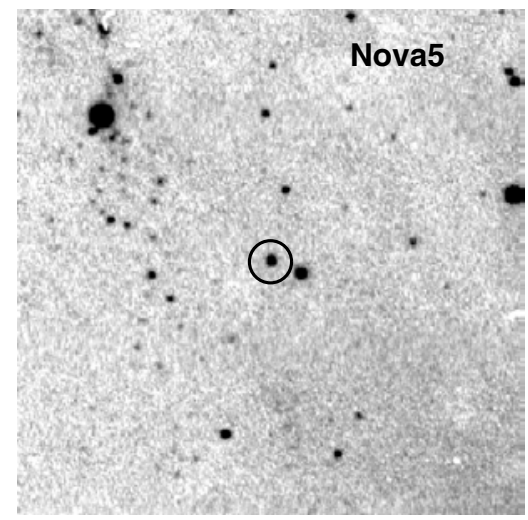

(e) Nova 5 (U)

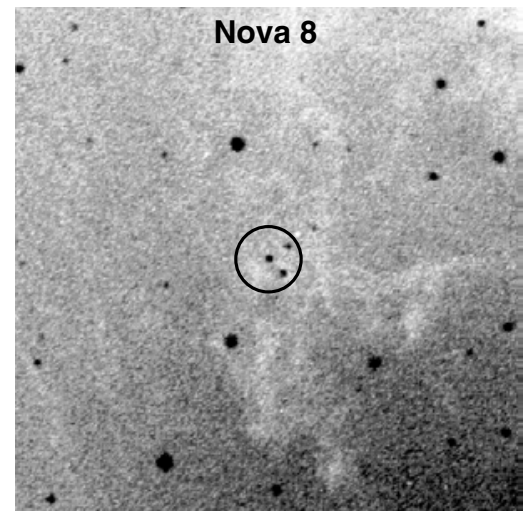

(h) Nova 8 (B)

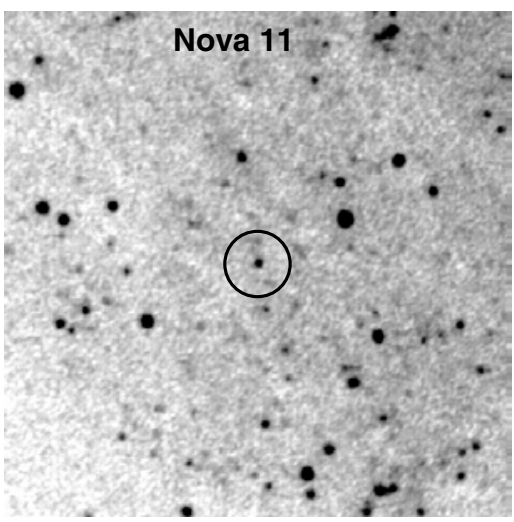

(k) Nova 11 (B)

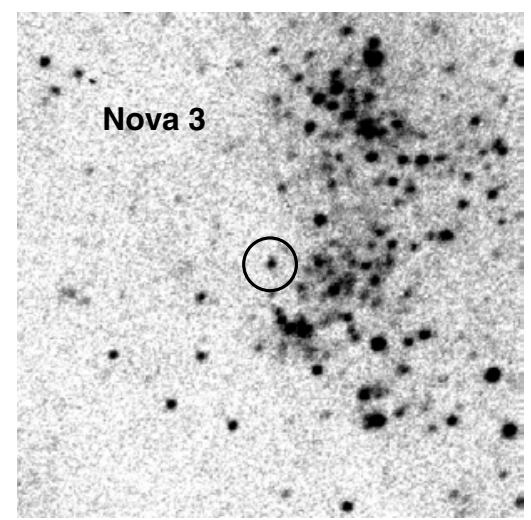

(c) Nova 3 (U)

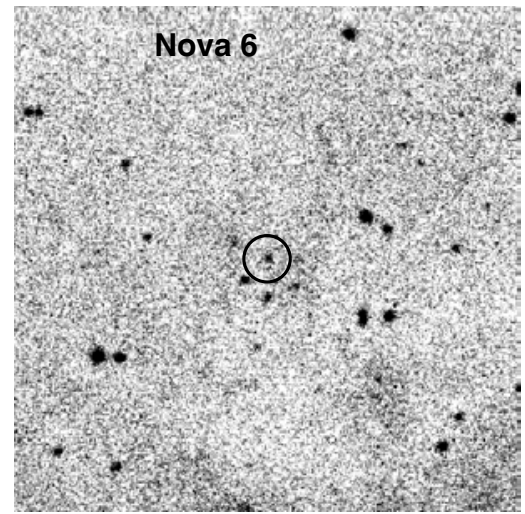

(f) Nova 6 (V)

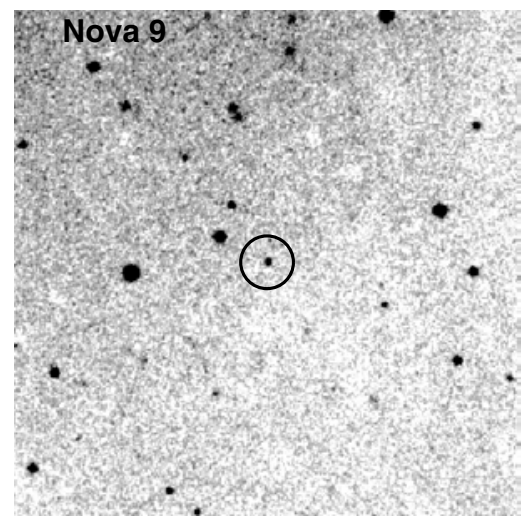

(i) Nova 9 (V)

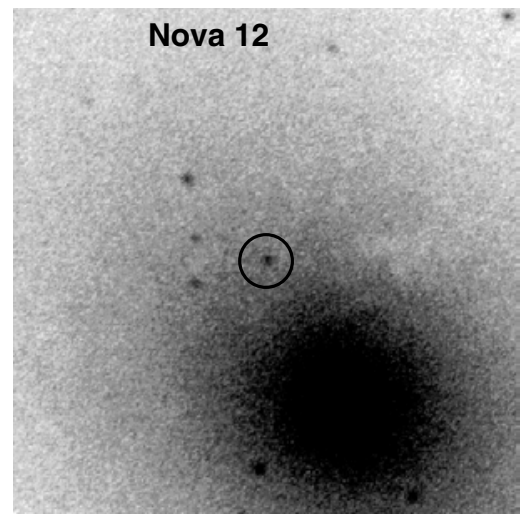

(l) Nova 12 (U)

Fig. 8. Finding charts for novae 1-12. 
M. Henze et al.: Search for novae in M31 on digitized Schmidt plates, Online Material p 11

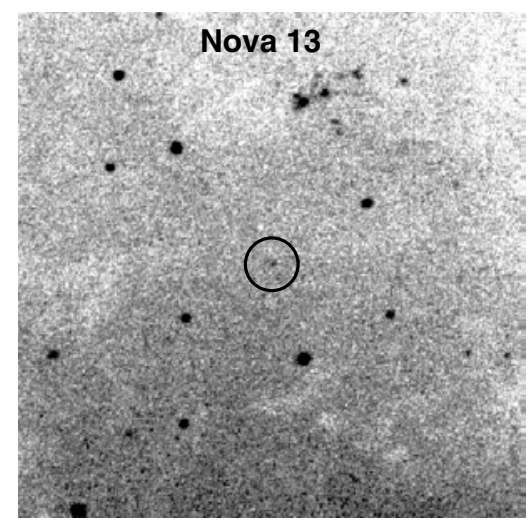

(a) Nova 13 (U)

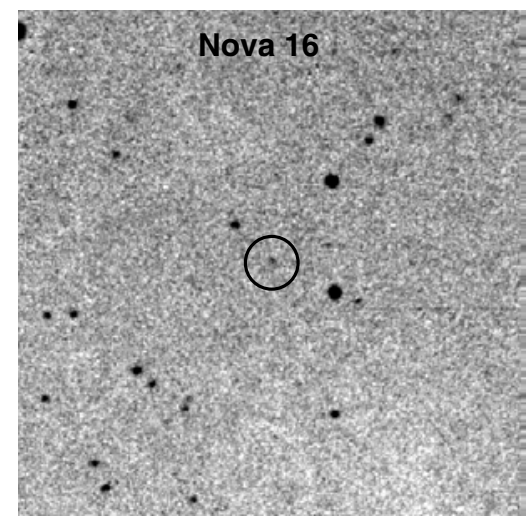

(d) Nova 16 (U)

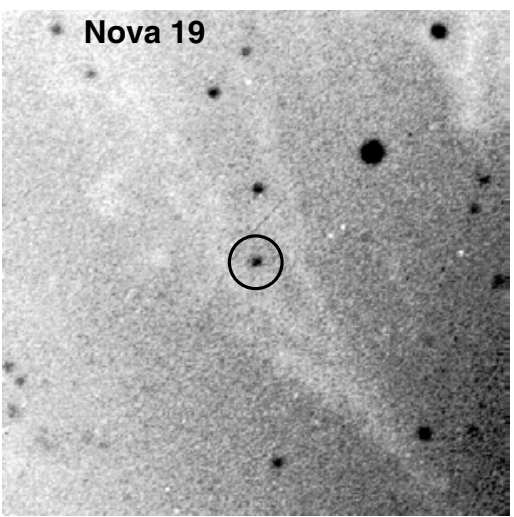

(g) Nova 19 (B)

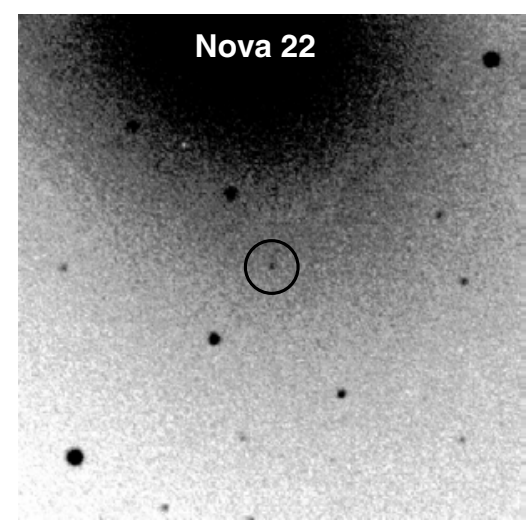

(j) Nova $22(B)$

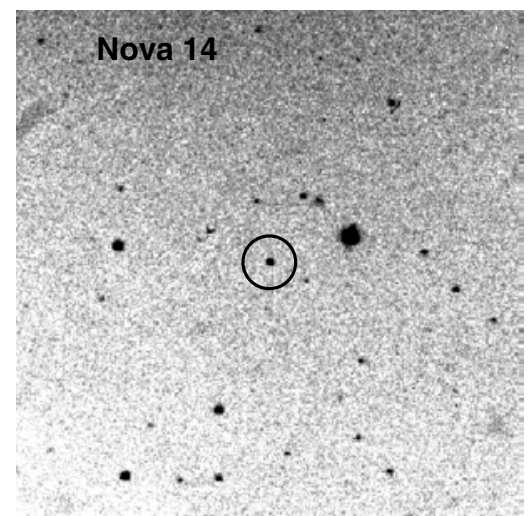

(b) Nova 14 (U)

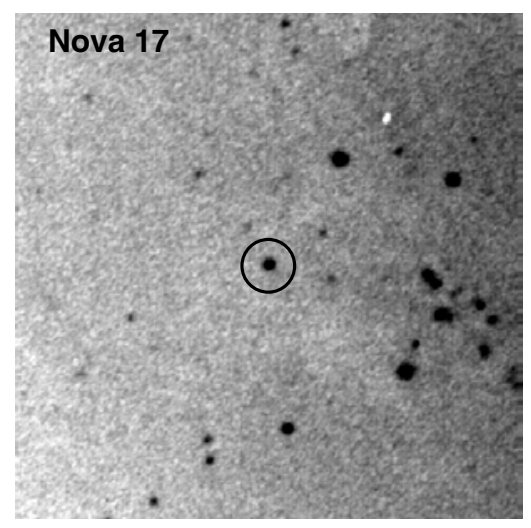

(e) Nova 17 (B)

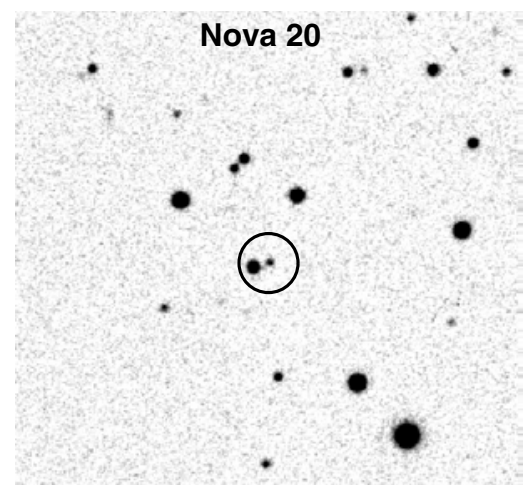

(h) Nova 20 (B)

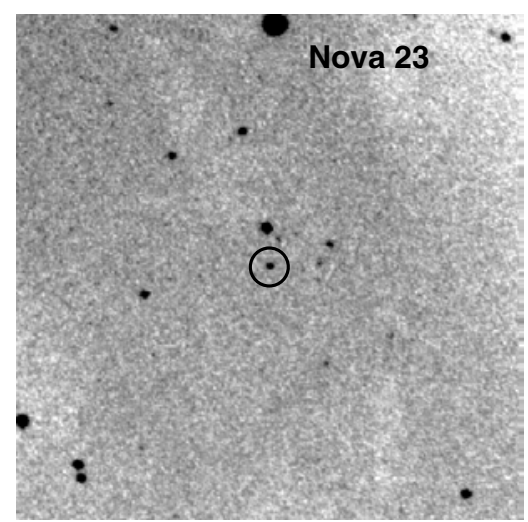

(k) Nova 23 (B)

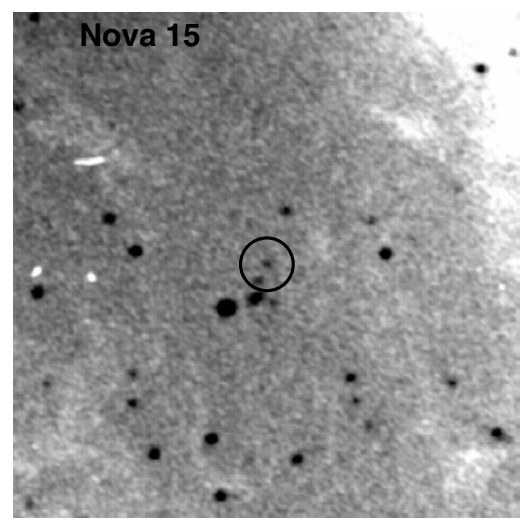

(c) Nova 15 (B)

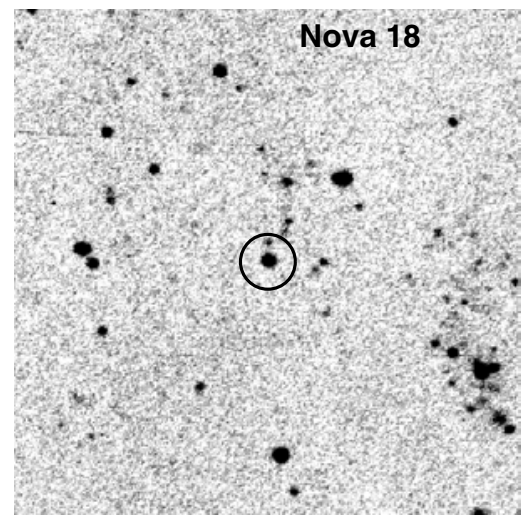

(f) Nova 18 (U)

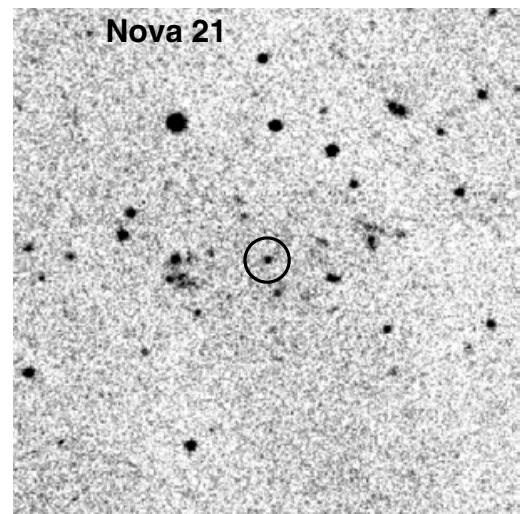

(i) Nova 21 (B)

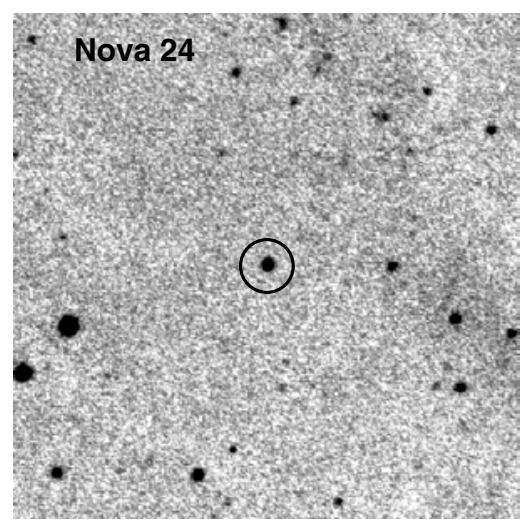

(1) Nova 24 (B)

Fig. 9. Finding charts for novae 13-24. 
M. Henze et al.: Search for novae in M31 on digitized Schmidt plates, Online Material p 12

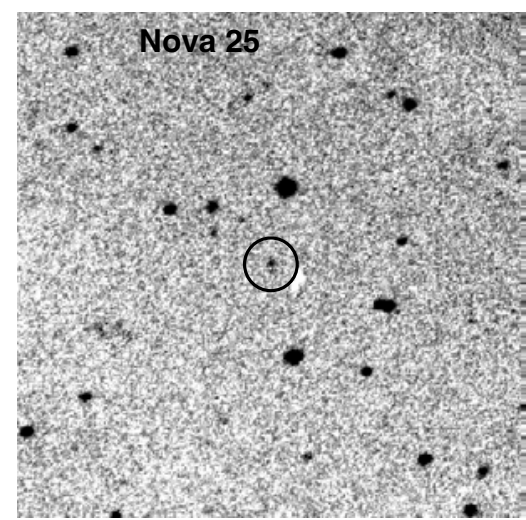

(a) Nova 25 (U)

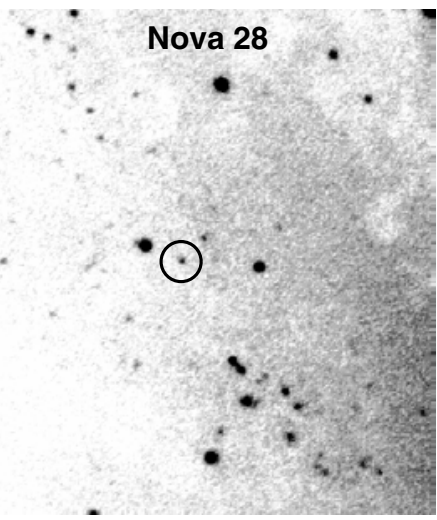

(d) Nova 28 (B)

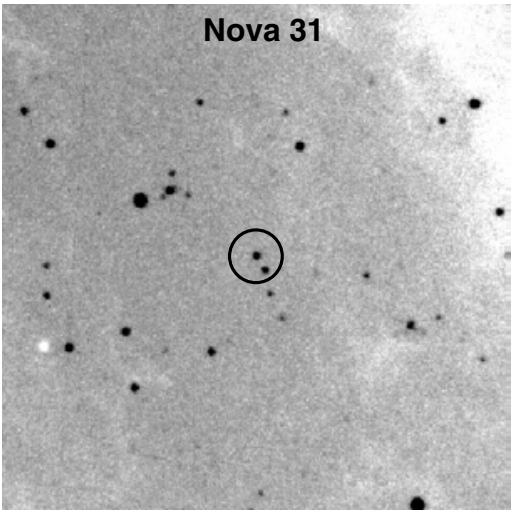

(g) Nova 31 (B)

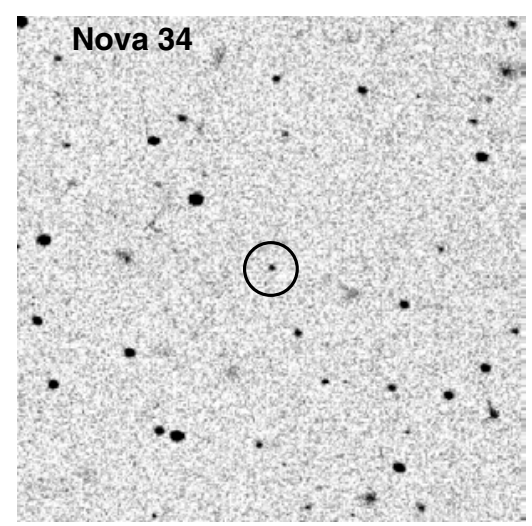

(j) Nova 34 (B)

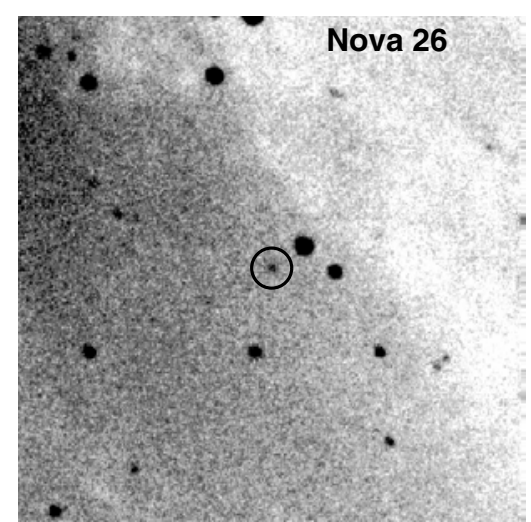

(b) Nova 26 (B)

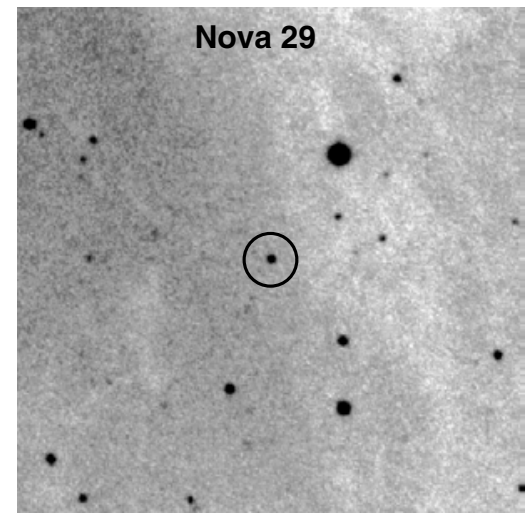

(e) Nova 29 (B)

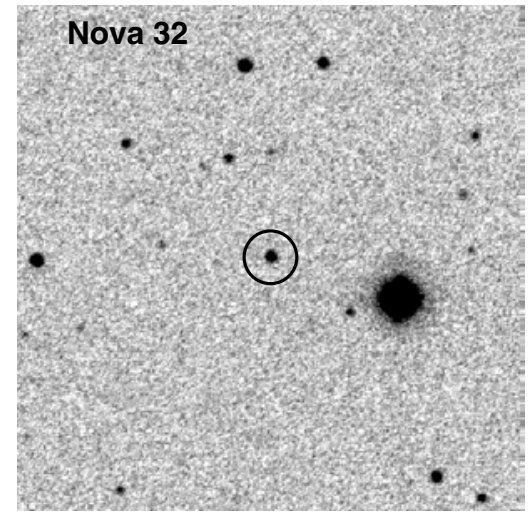

(h) Nova $32(\mathrm{~B})$

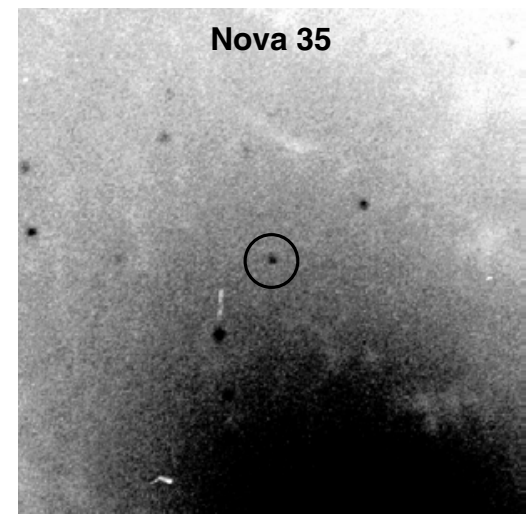

(k) Nova 35 (U)

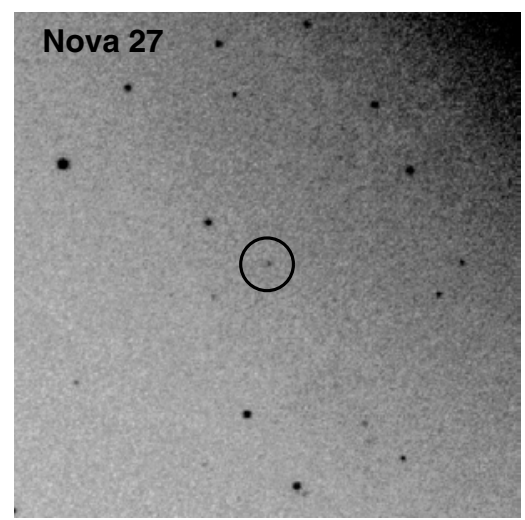

(c) Nova 27 (B)

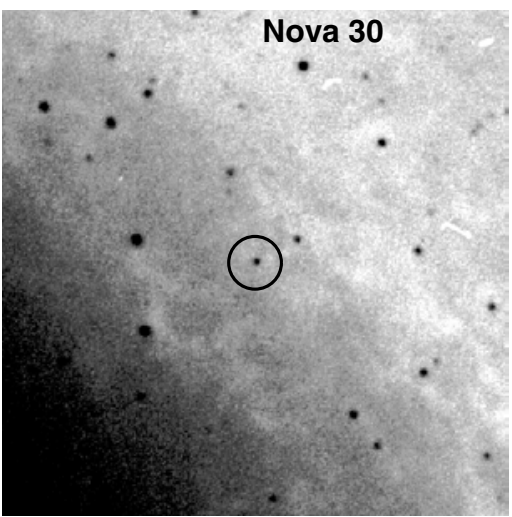

(f) Nova 30 (B)

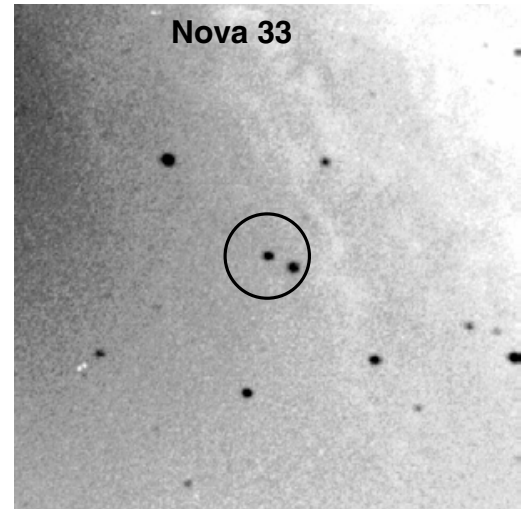

(i) Nova 33 (B)

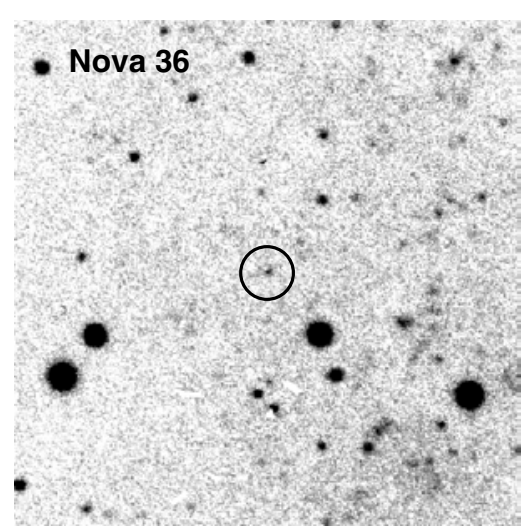

(1) Nova 36 (U)

Fig. 10. Finding charts for novae 25-36. 
M. Henze et al.: Search for novae in M31 on digitized Schmidt plates, Online Material $p 13$

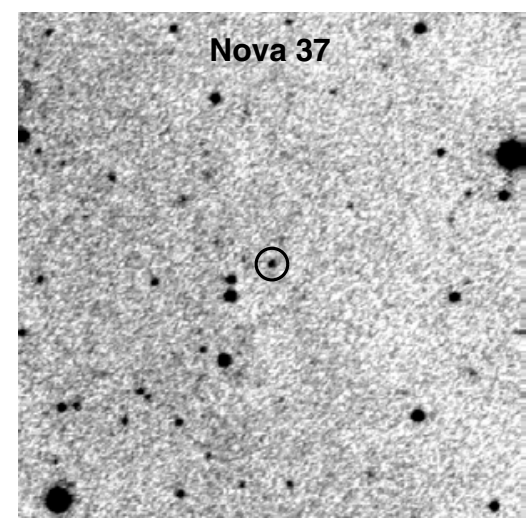

(a) Nova 37 (V)

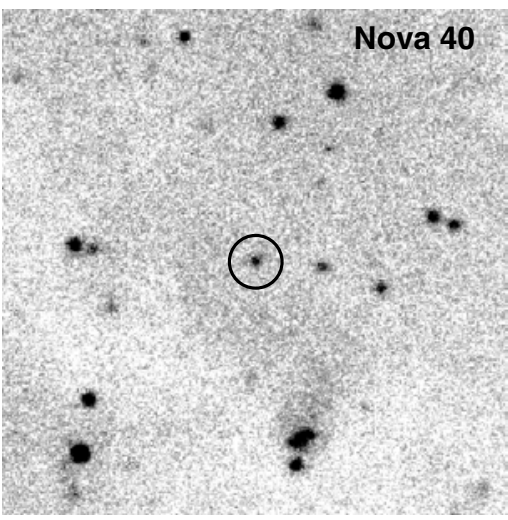

(d) Nova 40 (B)

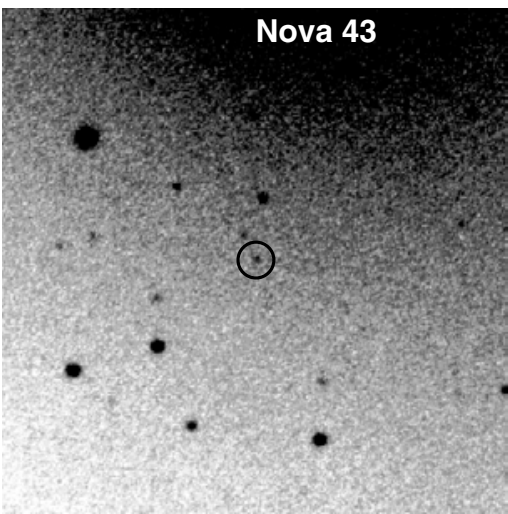

(g) Nova 43 (B)

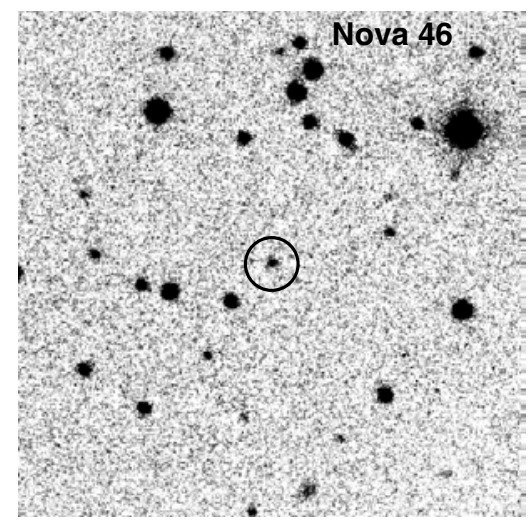

(j) Nova 46 (B)

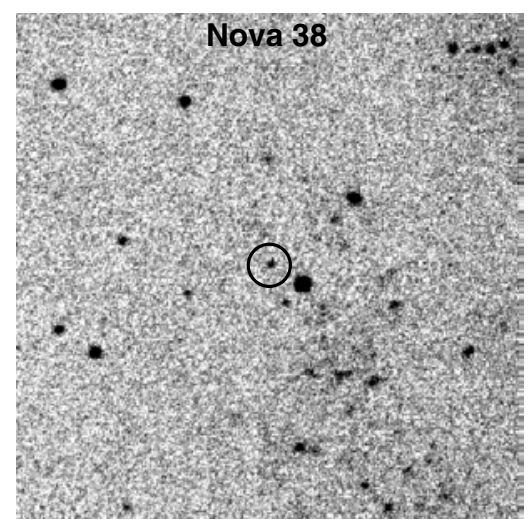

(b) Nova 38 (B)

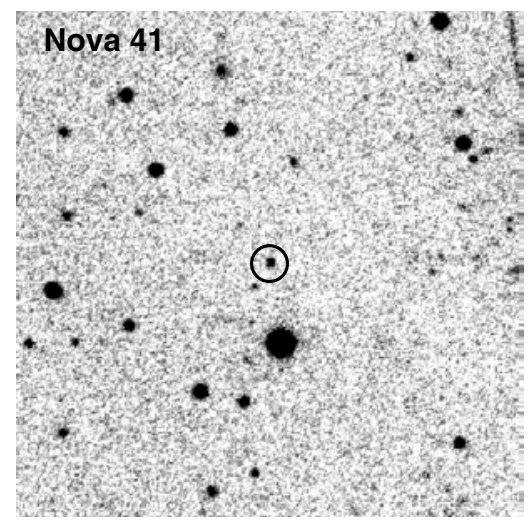

(e) Nova 41 (B)

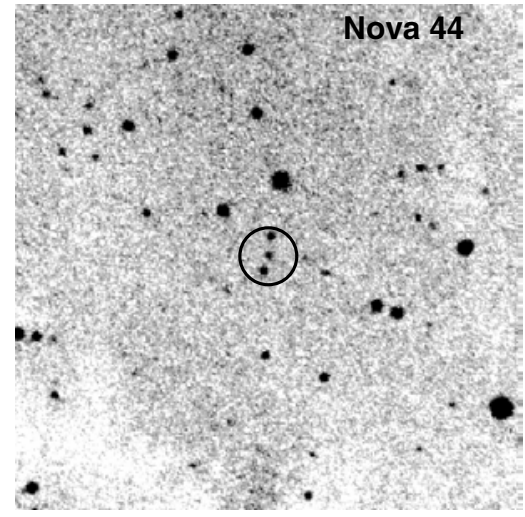

(h) Nova $44(\mathrm{~V})$

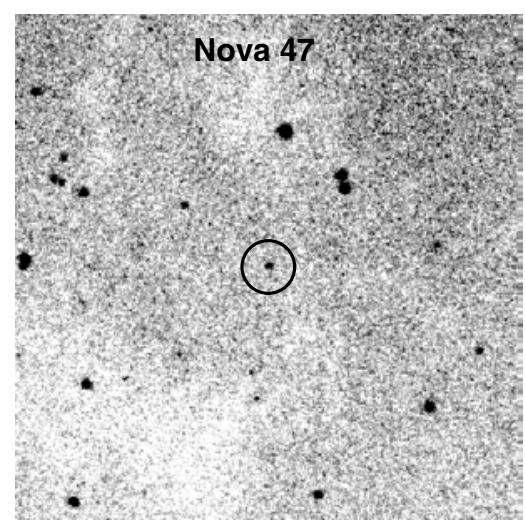

(k) Nova 47 (B)

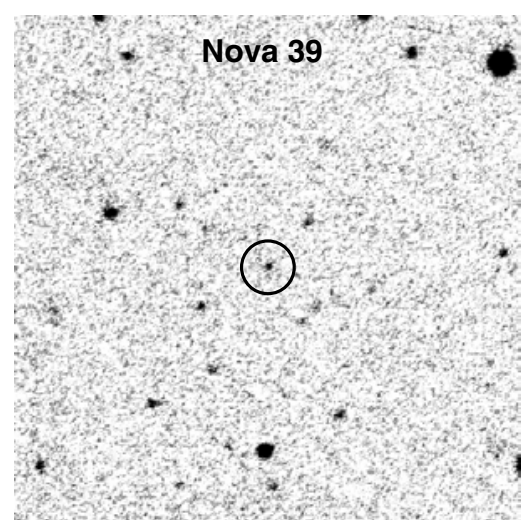

(c) Nova 39 (B)

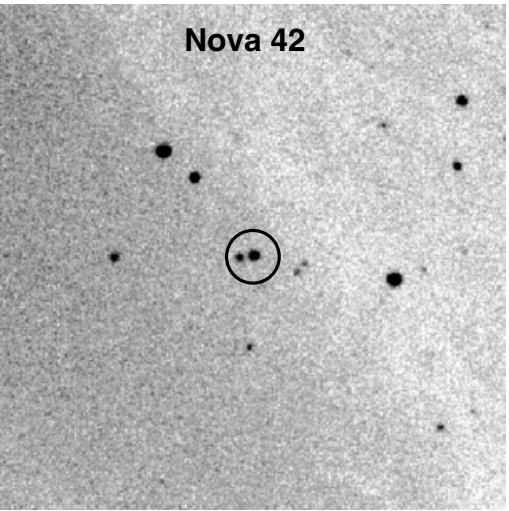

(f) Nova 42 (B)

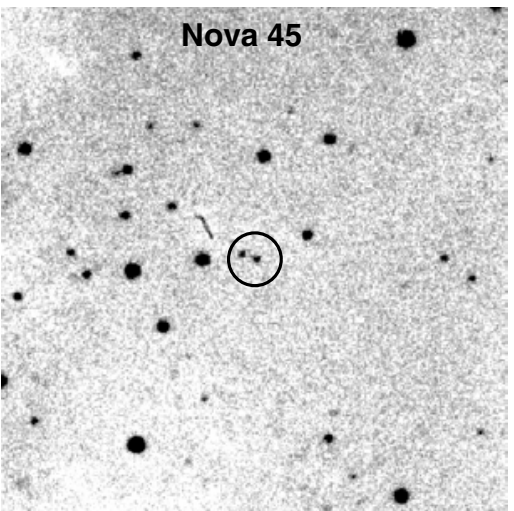

(i) Nova 45 (B)

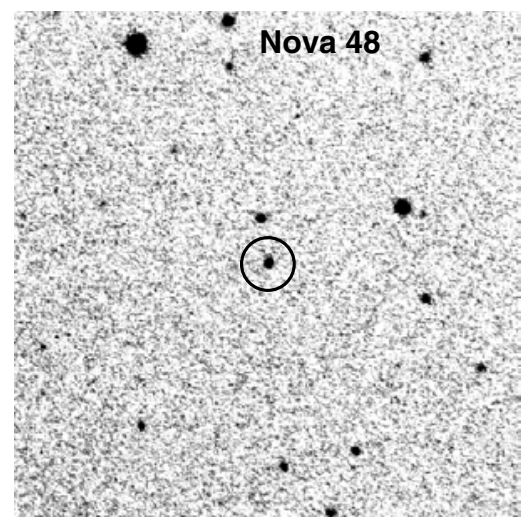

(1) Nova 48 (B)

Fig. 11. Finding charts for novae 37-48. 
M. Henze et al.: Search for novae in M31 on digitized Schmidt plates, Online Material p 14

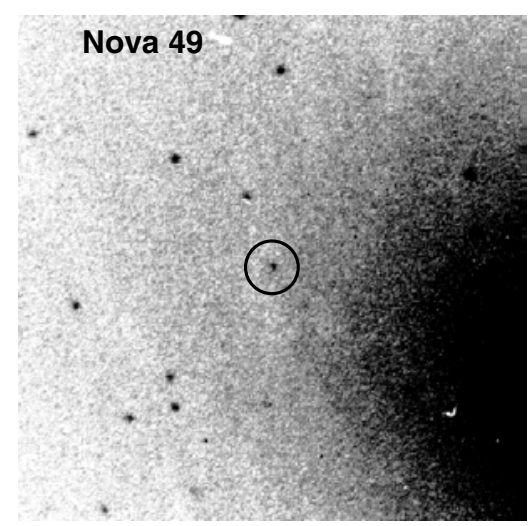

(a) Nova 49 (U)

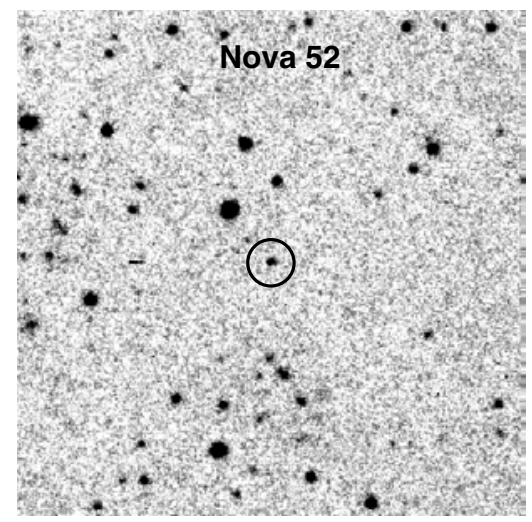

(d) Nova 52 (B)

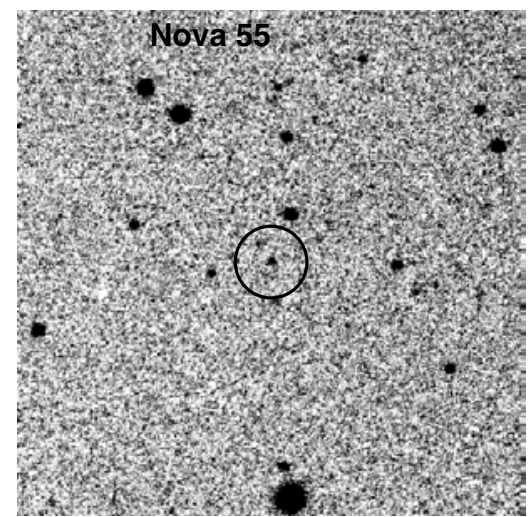

(g) Nova 55 (B)

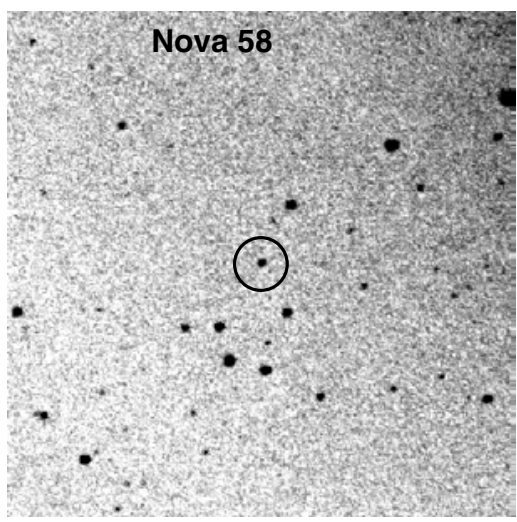

(j) Nova $58(\mathrm{U})$

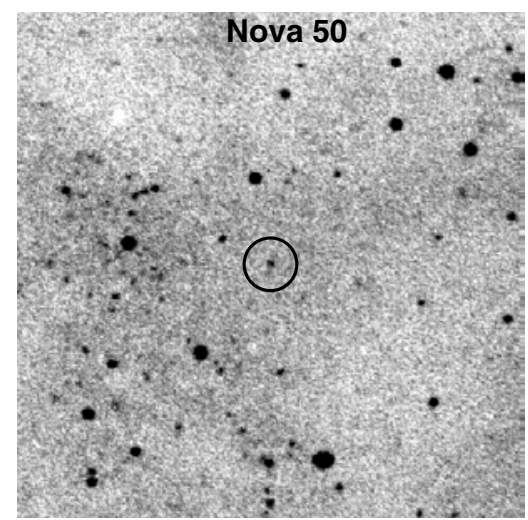

(b) Nova 50 (B)

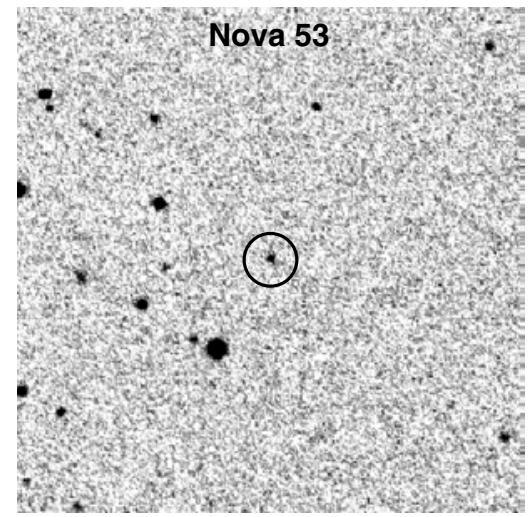

(e) Nova 53 (U)

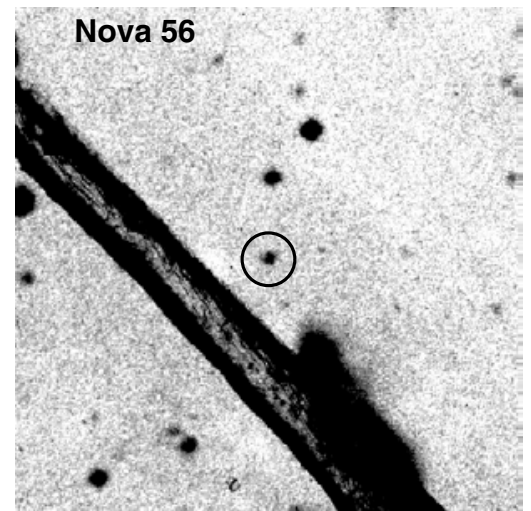

(h) Nova 56 (B)

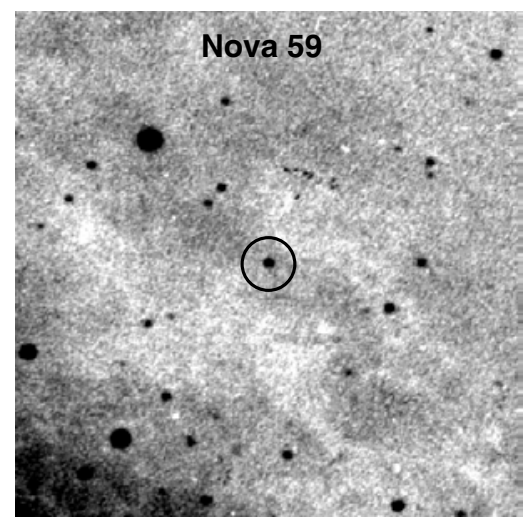

(k) Nova 59 (B)

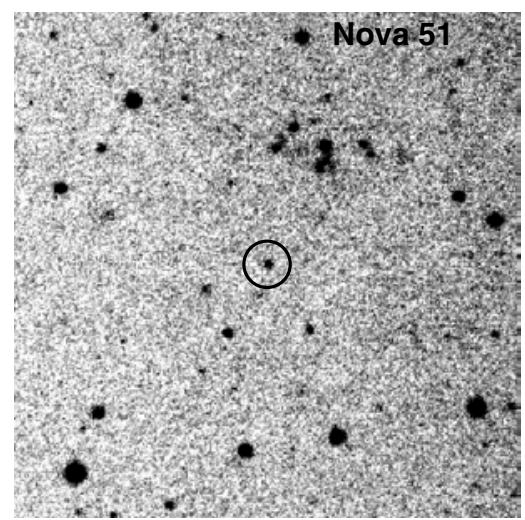

(c) Nova 51 (B)

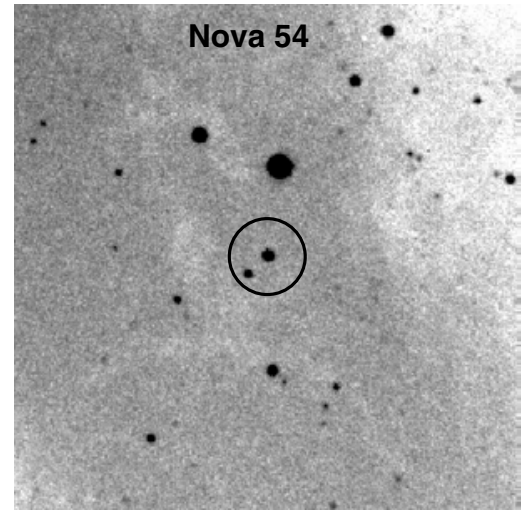

(f) Nova 54 (B)

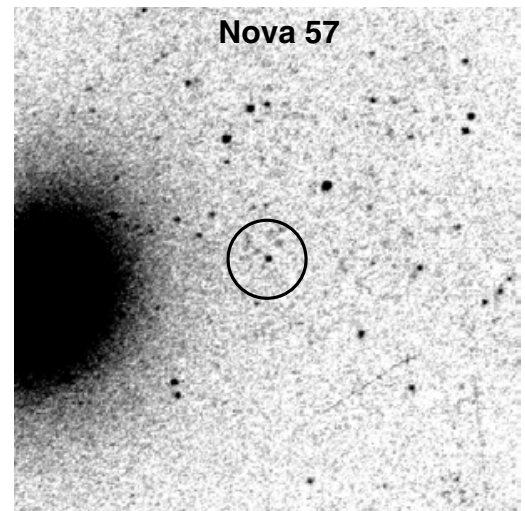

(i) Nova 57 (U)

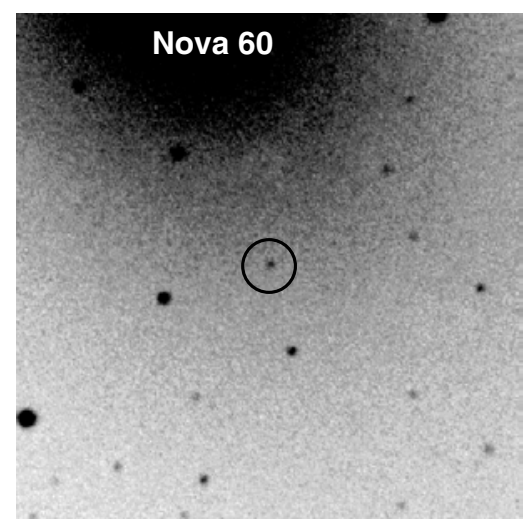

(1) Nova 60 (U)

Fig. 12. Finding charts for novae 49-60. 
M. Henze et al.: Search for novae in M31 on digitized Schmidt plates, Online Material p 15

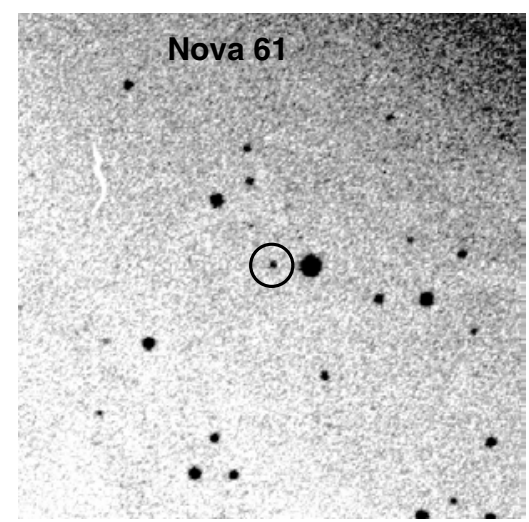

(a) Nova 61 (B)

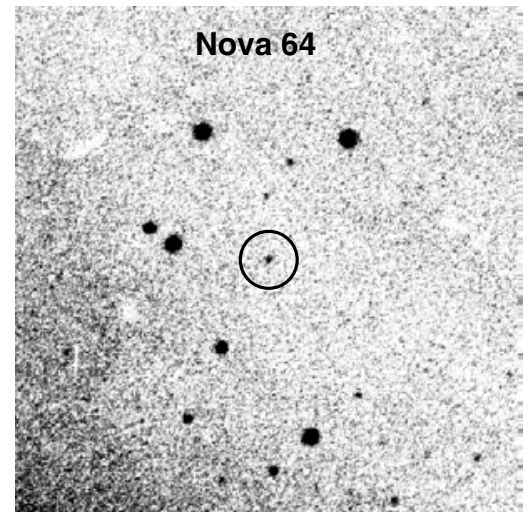

(d) Nova 64 (U)

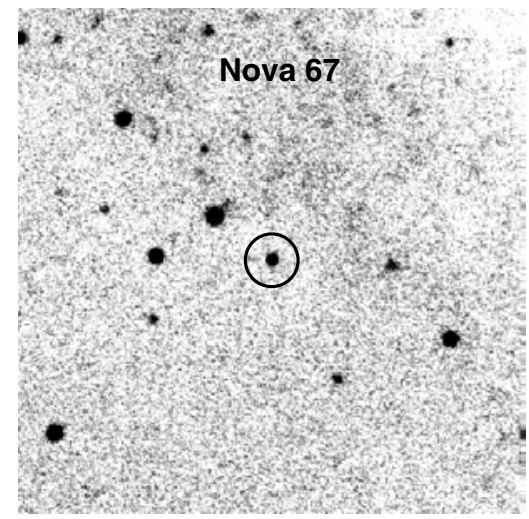

(g) Nova $67(\mathrm{~V})$

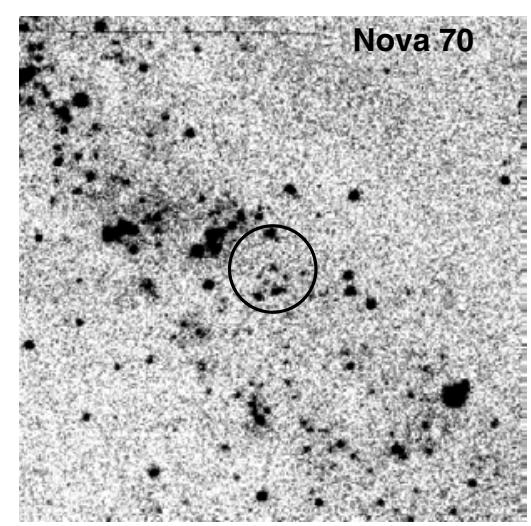

(j) Nova $70(\mathrm{U})$

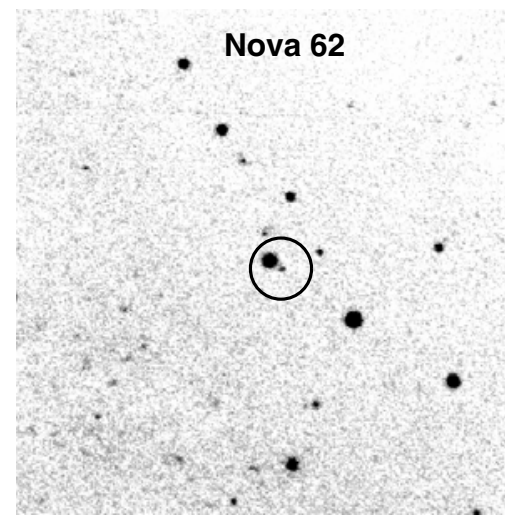

(b) Nova $62(\mathrm{~B})$

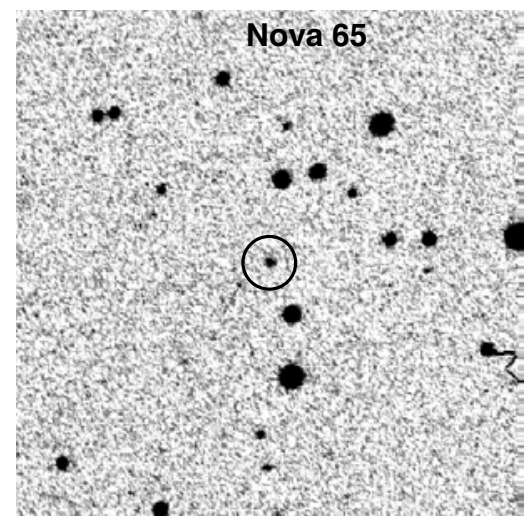

(e) Nova 65 (B)

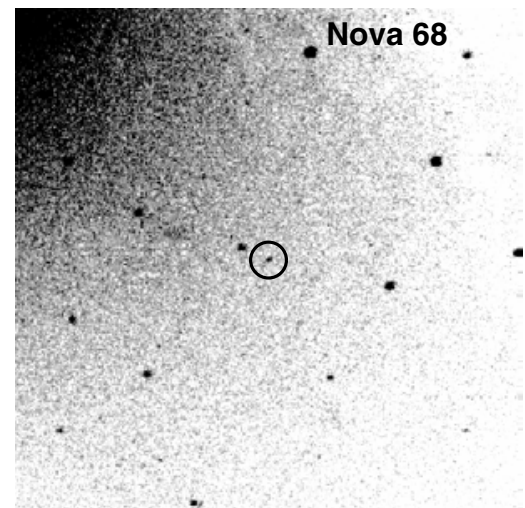

(h) Nova $68(\mathrm{U})$

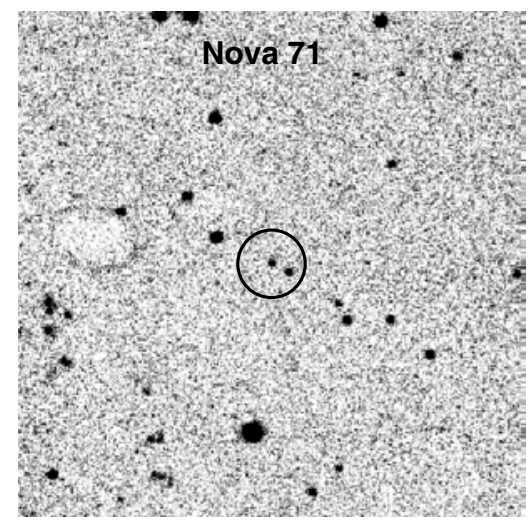

(k) Nova $71(\mathrm{U})$

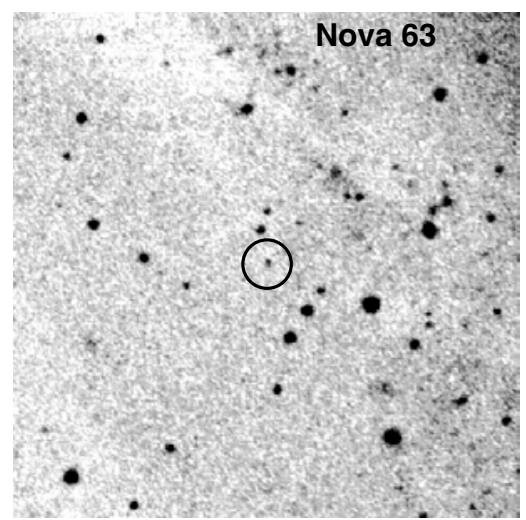

(c) Nova 63 (B)

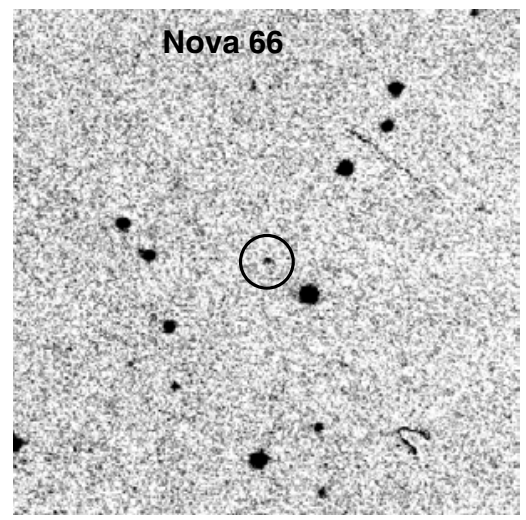

(f) Nova 66 (U)

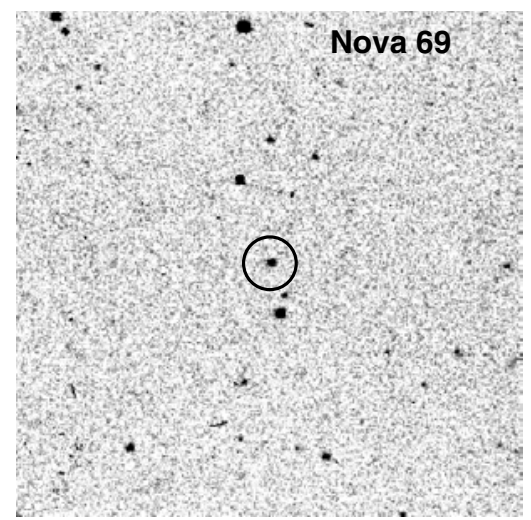

(i) Nova 69 (U)

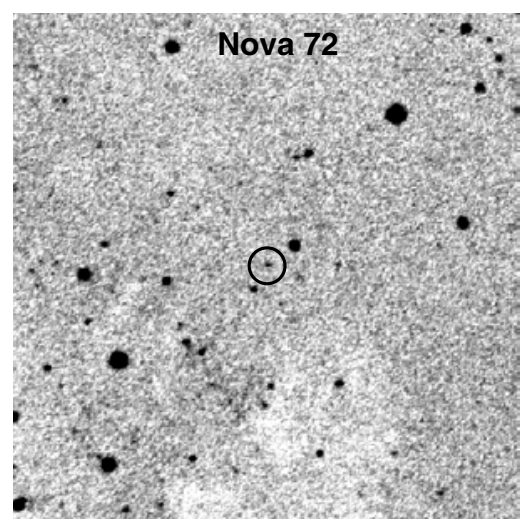

(1) Nova 72 (B)

Fig. 13. Finding charts for novae 61-72. 
M. Henze et al.: Search for novae in M31 on digitized Schmidt plates, Online Material p 16

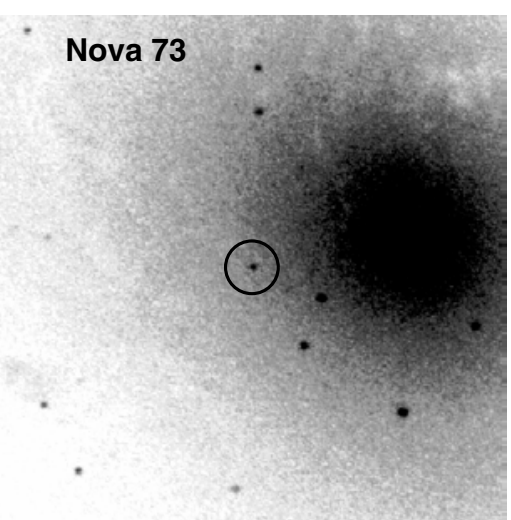

(a) Nova 73 (U)

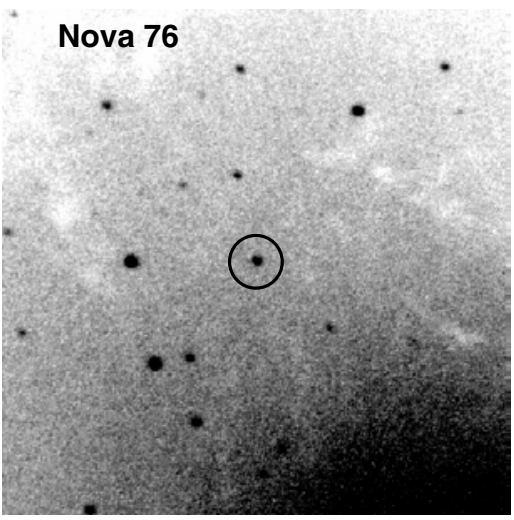

(d) Nova 76 (B)

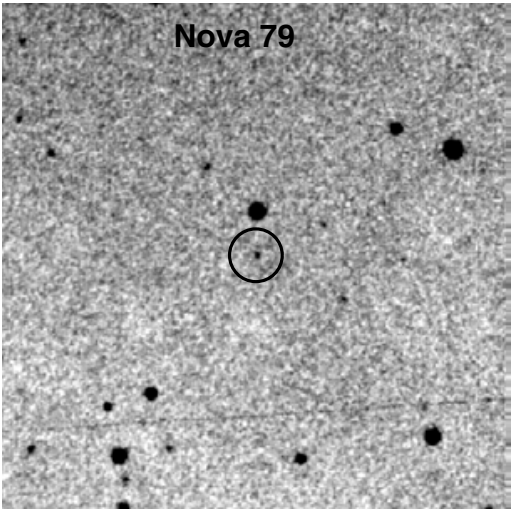

(g) Nova $79(\mathrm{U})$

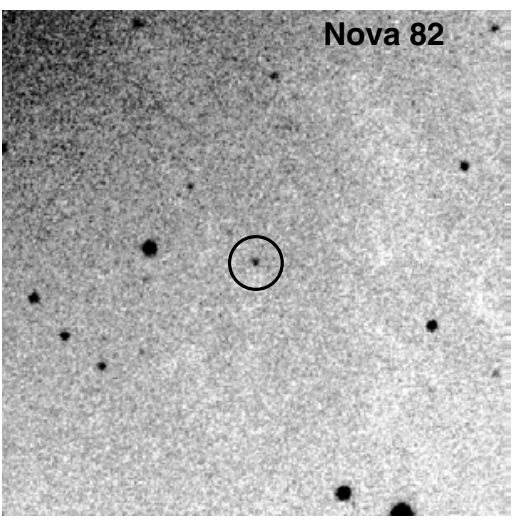

(j) Nova $82(\mathrm{U})$

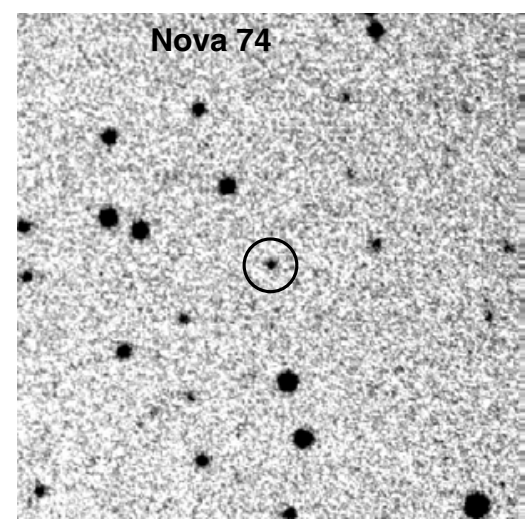

(b) Nova 74 (B)

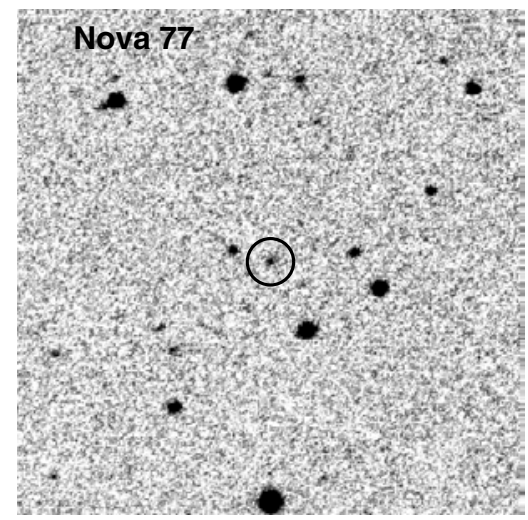

(e) Nova $77(\mathrm{U})$

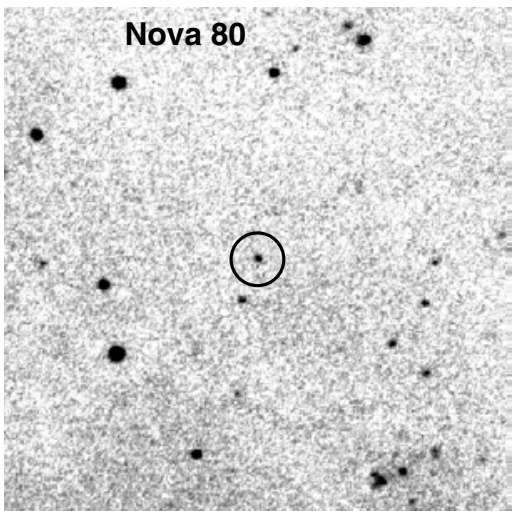

(h) Nova $80(\mathrm{U})$

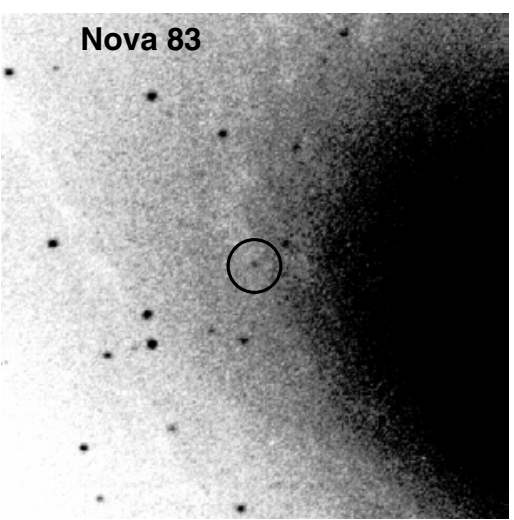

(k) Nova 83 (B)

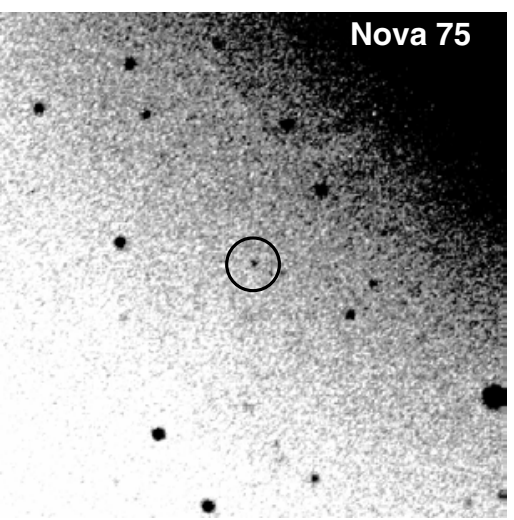

(c) Nova 75 (B)

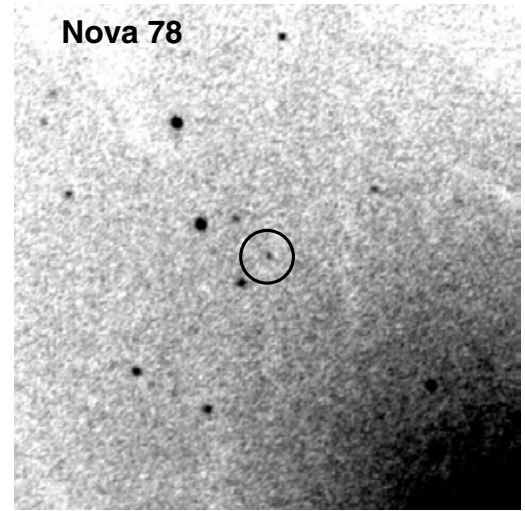

(f) Nova 78 (U)

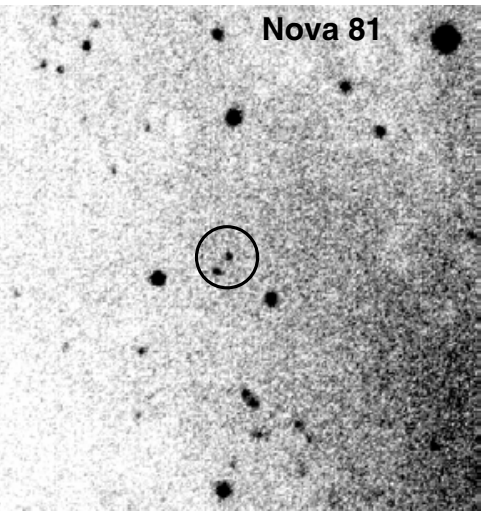

(i) Nova $81(\mathrm{~V})$

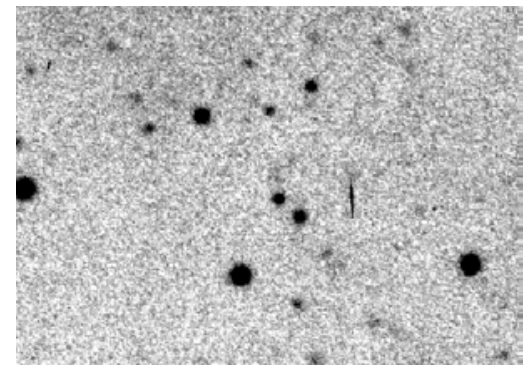

